\title{
BMO ON STRONGLY PSEUDOCONVEX DOMAINS: HANKEL OPERATORS, DUALITY AND $\bar{\partial}$-ESTIMATES
}

\author{
HUIPING LI AND DANIEL H. LUECKING
}

\begin{abstract}
We study the condition that characterizes the symbols of bounded Hankel operators on the Bergman space of a strongly pseudoconvex domain and show that it is equivalent to $B M O$ plus analytic. (Here we mean the Bergman metric $B M O$ of Berger, Coburn and Zhu.) In the course of the proof we obtain new $\bar{\partial}$-estimates that may be of independent interest. Some applications include a decomposition of $B M O$ similar to the classical $L^{\infty}+\widetilde{L^{\infty}}$, and two characterizations of the dual of $V M O$ (which is also a predual of $B M O$ ).

In addition, we obtain some partial results on the boundedness of Hankel operators in $L^{1}$ norm.
\end{abstract}

\section{INTRODUCTION}

Let $D$ be a strongly pseudoconvex domain in $\mathbb{C}^{n}$, and let $d V$ denote $2 n$ dimensional volume measure on $D$. (The reader may assume on first reading that $D$ is $\mathbb{D}$, the open unit disk, substituting area measure $d A$ for $d V$.) Let $L^{2}=L^{2}(d V)$ denote the space of square integrable measurable functions on $D$, $\mathscr{H}$ the space of all holomorphic functions on $D$, and $A^{2}=L^{2} \cap \mathscr{H}$. Denote by $P$ the orthogonal projection from $L^{2}$ onto $A^{2}$, which is effected by integrating against the Bergman kernel $K=K_{D}(z, w)$. Define the Hankel operator $H_{f}$ with symbol $f$ by

$$
H_{f} g=f g-P(f g)
$$

where $f$ is some $L^{1}$ function on $D$. This is defined (via the integral formula for $P$ ) whenever $g$ is a bounded analytic function on $D$. Moreover, if $h \in$ $A^{1}=L^{1} \cap \mathscr{H}$, then $H_{h} g=0$ for all bounded analytic $g$. This allows $H_{f}$ to be densely defined for any $f \in L^{1}+\mathscr{H}$, and it is guaranteed to be defined on all of $A^{2}$ (and bounded) whenever $f$ is bounded. It may, however, extend to be a bounded linear operator on $A^{2}$ (or even $A^{p}, 1<p<\infty$ ) even when $f$ is not bounded.

Necessary and sufficient conditions for the extension of $H_{f}$ to a bounded operator from $A^{p}$ to $L^{p}$ were obtained in [Lu3] for the unit disk, and these results were extended to strongly pseudoconvex domains and other settings in [Li2]. The conditions are formally the same in the two settings and will be discussed in more detail below. For now, let us say that the condition for $H_{f}$ to be bounded from $A^{p}$ to $L^{p}$ is a uniform bound on the distance from $\left.f\right|_{B(z)}$

Received by the editors April 15, 1994.

1991 Mathematics Subject Classification. Primary 47B38; Secondary 32A37.

Key words and phrases. Hankel operator, $B M O$, strongly pseudoconvex domain. 
to analytic functions, where $B(z)$ is a ball in the Bergman metric (of some fixed size) centered at $z$, and the distance is taken in the $L^{p}$ norm with respect to normalized Lebesgue measure on $B(z)$. We informally termed this property the $B D A$ condition (for "Bounded Distance to Analytic") but did not formally define a space of functions based on it, perhaps because we were convinced it had a better characterization.

Part of the purpose of this paper is to present such a better characterization. If we note that the space $B M O$ of Berger, Coburn, and $\mathrm{Zhu}[\mathrm{BCZ}]$ is precisely the space with "bounded distance to constants", it is clear that $B M O+\mathscr{H}$ is contained in $B D A$. Our main purpose here is to prove the opposite containment and to explore some of the implications of this fact and of its proof. These implications include a representation of $B M O$ similar to the " $L^{\infty}+\widetilde{L^{\infty}}$ " representation of the classical $B M O$ on $\mathbb{R}^{n}$ and several representations of the predual of $B M O$ (which is also the dual of $V M O$ ). In addition, some partial results on the boundedness of Hankel operators from $A^{1}$ to $L^{1}$ will be presented, including a complete characterization for conjugate analytic symbol.

\section{BACKGROUND AND HISTORY}

The seminal paper on the boundedness of $H_{f}$ is S. Axler's [Ax2]. In that paper $f$ is assumed to be the conjugate of an analytic function $h$ in the unit disk $\mathbb{D}, f=\bar{h}$. The result obtained is that $H_{\bar{h}}$ extends to be a bounded operator from $A^{2}$ to $L^{2}$ if and only if $h$ is in the Bloch space $\mathscr{B}$, that is, $\left(1-|z|^{2}\right) h^{\prime}(z)$ is bounded in $\mathbb{D}$. Moreover, $H_{f}$ is compact if and only if $h \in \mathscr{B}_{0}$, that is, $\left(1-|z|^{2}\right) h^{\prime}(z) \rightarrow 0$ as $|z| \rightarrow 1$. Other results (also in [Ax2]) show that $\mathscr{B}$ is $\mathscr{H} \cap B M O$ and $\mathscr{B}_{0}$ is $\mathscr{H} \cap V M O$.

The next significant steps were taken by D. Békollé, C. Berger, L. Coburn and $\mathrm{K}$. Zhu in the two papers [BCZ] and [BBCZ]. In those papers the setting is a bounded symmetric domain in $\mathbb{C}^{n}$. They showed the following:

Theorem A (Békollé, Berger, Coburn, Zhu). The following are equivalent for a bounded symmetric domain.

(a) $H_{f}$ and $H_{\bar{f}}$ are simultaneously bounded.

(b) $f$ belongs to $B M O$.

Let us interrupt here to define $B M O$ and the related $V M O$. First, let $\beta$ denote the Bergman distance (the integrated form of the Bergman metric) on the bounded domain $D$. The symbol $D$ may now represent either a bounded symmetric domain or a strongly pseudoconvex domain. On the disk $\mathbb{D}, \beta$ is the same as the hyperbolic distance. Let $r>0$ be some fixed number and let $B(z)=B(z, r)=\{w \in D: \beta(z, w)<r\}$ denote the ball with respect to $\beta$ centered at $z$ with radius $r$. Finally, let the Lebesgue measure of a set $E$ be denoted by $|E|$.

2.1. Definition. Let $1<p<\infty$ and let $f$ be a measurable function on $D$ locally in $L^{p}$. The $p$-mean oscillation of $f$ at $z$ is defined to be

$$
M O_{p}(f, z)=\left(\inf _{c \in \mathbb{C}} \frac{1}{|B(z)|} \int_{B(z)}|f-c|^{p} d V\right)^{1 / p}
$$


We say that $f \in B M O_{p}$ (respectively, $f \in V M O_{p}$ ) if and only if

$$
\sup _{z \in D} M O_{p}(f, z)<\infty \quad\left(\text { respectively, } \lim _{z \rightarrow \dot{D} D} M O_{p}(f, z)=0\right)
$$

and we write $\|f\|_{B M O, p}$ for the supremum. If the subscript $p$ is omitted in any of the above notation then $p=2$ is assumed.

It is well known that the different choices for $r$ yield equivalent norms on $B M O_{p}$. Theorem A above is accompanied by a similar one in which "compact" replaces "bounded" and " $V M O$ " replaces " $B M O$ ".

Theorem $\mathrm{A}$ is equivalent to characterizing the bounded Hankel operators for real $f$ only. It also extends Axler's result, since $H_{\bar{f}}=0$ when $\bar{f}$ is analytic and $\mathscr{B}=B M O \cap \mathscr{H}([\mathrm{Ax} 2])$. Nevertheless, it does not yield a characterization of the boundedness of $H_{f}$ alone without additional restrictions on $f$.

Theorem A was extended to strongly pseudoconvex domains by the first author $(\mathrm{H} . \mathrm{Li})$ in [Li1] and independently by $\mathrm{F}$. Beatrous and Song-Ying $\mathrm{Li}$ (in $[\mathrm{BL}])$. Then in [Lu3] the second author (Luecking) was able to remove all restrictions on $f$ (at least for the unit disk), requiring only that $f$ be integrable. The resulting characterization utilized an approach that did not quite work in other settings. The difficulties were overcome in [Li2] by the first author. That paper extended the result to Hankel operators on the Bergman space of a strongly pseudoconvex domain in $\mathbb{C}^{n}$, but also extended the proof to all the usual weighted Bergman spaces and to the Fock space. A different method (from [Lu3]) was used for part of the proof, and this improved the result even in the unit disk. Unfortunately, the approach has not yet yielded a proof for bounded symmetric domains, so that Theorem A is still the best result known in that setting.

Before proceeding further, we should formally define a few of the terms we have already used.

2.2. Definition. A domain $D$ in $\mathbb{C}^{n}$ will be called strongly pseudoconvex if there is a strictly plurisubharmonic defining function $\rho$ for $D$. That is, $\rho$ is defined and smooth (i.e., $C^{\infty}$ ) in a neighborhood of $\bar{D}$, the Levi form for $\rho$ is strictly positive definite on $\bar{D}$, the differential $d \rho$ is nonvanishing on $\partial D$, and $D=\{z: \rho(z)<0\}$. The Bloch space $\mathscr{B}$ (respectively, the little Bloch space $\mathscr{B}_{0}$ ) of the strongly pseudoconvex domain $D$ is the collection of all functions $f$ holomorphic on $D$ satisfying

$$
\left.\sup _{z \in D}|\rho(z) \partial f(z)|<\infty \quad \text { (respectively, } \lim _{z \rightarrow \partial D} \rho(z) \partial f(z)=0\right) .
$$

The interpretation of "smooth" to mean " $C$ " can be replaced with the interpretation "as differentiable as necessary". We will not be concerned with the exact level of differentiability required.

It automatically follows from (1) that the components of $\partial f$ in the complex directions perpendicular to $\partial \rho$ are $O\left(|\rho|^{-1 / 2}\right.$ ) (see [KM]). Moreover, it remains true in strongly pseudoconvex domains, as in the unit disk, that $\mathscr{B}=\mathscr{H} \cap B M O_{p}$ and $\mathscr{B}_{0}=\mathscr{H} \cap V M O_{p}$ for all $p \geq 1$ (see [Li1]).

Let us turn to the characterizations obtained in [Lu3] and [Li2]. Recall that $r>0$ has been fixed and $B(z)=B(z, r)$. Fix also $p>1$ and measurable $f$ on $D$, and define the following local measurement of the distance to analytic 
functions:

$$
F(z)=\left(\inf _{h \in \mathscr{E}} \frac{1}{|B(z)|} \int_{B(z)}|f-h|^{p} d V\right)^{1 / p}
$$

The function $F(z)$ depends on $f$ and on $p$, but this will not be reflected in the notation. Of course, $F$ also depends on $r$, but this fact is inessential.

Theorem B. Let $D$ be a strongly pseudoconvex domain in $\mathbb{C}^{n}$ with strictly plurisubharmonic defining function $\rho$ (satisfying $d \rho \neq 0$ on $\partial D$ ), and let $f \in L^{1}(D)$. For $p>1$ the following are equivalent.

(a) $H_{f}$ extends to a bounded operator from $A^{p}$ to $L^{p}$.

(b) $F$ is bounded on $D$ for some (respectively, every) choice of $r$.

(c) $f=f_{1}+f_{2}$, where

$$
G(z) \stackrel{\text { def }}{=}\left(\frac{1}{|B(z)|} \int_{B(z)}\left|f_{1}\right|^{p} d V\right)^{1 / p}
$$

is bounded on $D$ for some (respectively, every) choice of $r>0$ and $f_{2}$ is a $C^{\infty}$ function such that

$$
|\rho(z)|\left|\bar{\partial} f_{2}(z)\right|+|\rho(z)|^{1 / 2}\left|\bar{\partial} f_{2}(z) \wedge \bar{\partial} \rho(z)\right|
$$

is also bounded on $D$.

In the unit disk $\rho(z)=|z|^{2}-1$, the first term in (3) is $\left(1-|z|^{2}\right) \partial f_{2} / \partial \bar{z}$, and the second term is always 0 . Thus $\bar{\partial} f_{2}$ grows at most inversely as the distance to the boundary. This is also the case in a strongly pseudoconvex domain, but there the second term imposes the additional condition that the component of $\bar{\partial} f_{2}$ "parallel to the boundary" grows inversely as the square root of the distance to the boundary. The combination of derivatives in (3) appears in [V, Part 2] in connection with the study of $B M O$ (on the boundary) and the $\bar{\partial}$-equation.

Condition (b) of the theorem will be referred to as the " $B D A$ condition", and it will be convenient to define now the space $B D A_{p}$ :

2.3. Definition. Let $f$ be a measurable function on $D$ and define $F$ as in (2). If $F$ is bounded, we say that $f$ is in $B D A_{p}$.

The condition $f \in B D A_{p}$ clearly implies that $f$ is locally in $L^{p}$. Moreover, the implication (b) $\Rightarrow$ (c) of Theorem $B$ does not actually require the prior assumption that $f \in L^{1}$, and the proof of the implication (c) $\Rightarrow$ (a) shows that if $f \in B D A_{p}$ then $f \in L^{p}+\mathscr{H}$. There is an obvious seminorm $\|f\|_{B D A, p}=$ $\sup _{z \in D} F(z)$ on $B D A_{p}$ which makes $B D A_{p} / \mathscr{H}$ into a Banach space. The following is our improvement of the $B D A$ condition.

\subsection{Theorem. For $p \geq 1, B D A_{p}=B M O_{p}+\mathscr{H}$.}

We will prove this in $\S 3$ (delaying the proof of the main lemma until $\S 6$ ). In $\S 4$ we will explore some of the implications of this theorem and of its proof on the predual of $B M O_{p}$. In $\S 5$ we will consider some partial results on the boundedness of Hankel operators in the $L^{1}$ norm.

\section{THE MAIN THEOREM}

Let us begin by recalling a brief sketch of the proof of $(c) \Rightarrow$ (a) in Theorem B. Observe that for $f \in C^{1}$ and $g$ analytic, $H_{f} g$ is the minimal $L^{2}$ norm 
solution of the $\bar{\partial}$-equation

$$
\bar{\partial} u=g \bar{\partial} f .
$$

That is, $H_{f} g=(I-P) u$, where $u$ is any solution of (4). A class of kernels that solved the $\bar{\partial}$-equation in a strongly pseudoconvex domain was described in [BA]. A particular one of these $L(z, w)$ was constructed in [Li2]. $L(z, w)$ is an $(n, n-1)$-form in $w$ such that $u(z)=\int \varphi(z) \wedge L(z, w)$ solves $\bar{\partial} u=\varphi$ for any $\bar{\partial}$-closed $(0,1)$-form $\varphi$ which satisfies

$$
|\rho \varphi|+|\rho|^{1 / 2}|\varphi \wedge \bar{\partial} \rho| \in L^{1} .
$$

Moreover, it was shown in [Li2] that if the expression in (5) is bounded then the kernel $\varphi(w) \wedge L(z, w)$ defines a bounded operator on $L^{p}, p>1$. Since also $I-P$ is bounded on $L^{p}$, this result and condition (c) of Theorem B imply that $H_{f_{2}} g=(I-P) \int g(w) \bar{\partial} f_{2}(w) \wedge L(\cdot, w)$ is bounded from $A^{p}$ to $L^{p}$.

In order to prove Theorem 2.4 we will construct another such kernel $L$ and show that the same condition (the boundedness of (5) above) implies that $\varphi(w) \wedge L(z, w)$ defines a bounded map from $L^{\infty}$ to $B M O_{p}$. Having accomplished this we can finish the proof in short order as follows: If $f \in B D A_{p}$ then by the $(\mathrm{b}) \Rightarrow$ (c) portion of Theorem B there is a decomposition $f=f_{1}+f_{2}$. The condition (c) on $f_{1}$ clearly places it in $B M O_{p}$. Applying the operator with kernel $\bar{\partial} f_{2}(w) \wedge L(z, w)$ to the function 1 gives an element $u$ of $B M O_{p}$ that solves $\bar{\partial} u=\bar{\partial} f_{2}$. Then $f_{2}-u \in \mathscr{H}$ and so $f=\left(f_{1}+u\right)+\left(f_{2}-u\right) \in B M O_{p}+\mathscr{H}$. We will actually show somewhat more. The solution $u$ will be shown to have bounded oscillation rather than just bounded mean oscillation, and, by showing that $P$ is bounded from $B M O_{p}$ to $\mathscr{B}$, we will see that if $f \in L^{1}$ and $H_{f}$ is bounded on $A^{p}$ then $(I-P) f \in B M O_{p}$. (One minor point we ought to note is that the $p=1$ version of this argument is valid: As will be remarked in Theorem 5.1, the forward implications $(a) \Rightarrow(b) \Rightarrow(c)$ of Theorem B follow in the same way for $p=1$ as for $p>1$, and Proposition 3.2 will show that $P$ is bounded also from $B M O_{1}$ to $\mathscr{B}$.)

In the case of the unit disk, the minimal $L^{2}$ norm solution to the $\bar{\partial}$-equation $\bar{\partial} u=\varphi d \bar{z}$ is

$$
u(z)=\frac{1}{2 \pi i} \int_{\mathbb{D}} \frac{1-|w|^{2}}{(z-w)(1-z \bar{w})} \varphi(w) d \bar{w} \wedge d w .
$$

Following the above approach, $H_{f_{2}} g$ is given by

$$
H_{f_{2}} g(z)=\frac{1}{2 \pi i} \int_{\mathbb{D}} \frac{g(w)\left(1-|w|^{2}\right) \bar{\partial} f_{2}(w)}{(z-w)(1-z \bar{w})} \wedge d w .
$$

Letting $T(z, w)=[2 \pi(z-w)(1-z \bar{w})]^{-1}$, it is straightforward to prove that $T$ defines a bounded operator on the $L^{p}$ spaces. This is the (nearly trivial) unit disk version of the work in [Li2]. Given the explicit nature of the kernel $T$, it is another straightforward exercise to show that it takes bounded functions to $B M O_{p}$. In fact it is easy to show more, namely that $T$ takes bounded functions to functions $u$ that satisfy

$$
\left|u\left(z_{1}\right)-u\left(z_{2}\right)\right| \leq C\left(1+\beta\left(z_{1}, z_{2}\right)\right)
$$

with $C$ independent of $z_{1}$ and $z_{2}$. We will obtain a similar result for strongly pseudoconvex domains. 
3.1. Lemma. Let $\varphi$ be a $\bar{\partial}$-closed $(0,1)$-form satisfying

$$
|\rho \varphi|+|\rho|^{1 / 2}|\varphi \wedge \bar{\partial} \rho| \text { is bounded } .
$$

Then there is a solution $u$ to the equation $\bar{\partial} u=\varphi$ which has the form $u=$ $u_{1}+u_{2}$, where

$$
\left\|u_{1}\right\|_{\infty}+\left\|\rho \nabla u_{2}\right\|_{\infty} \leq C\left\||\rho \varphi|+|\rho|^{1 / 2}|\varphi \wedge \bar{\partial} \rho|\right\|_{\infty}
$$

and

$$
\left|\partial u_{2}(z) \wedge \partial \rho(z)\right|+\left|\bar{\partial} u_{2}(z) \wedge \bar{\partial} \rho(z)\right| \leq C|\rho(z)|^{-1 / 2}\left\||\rho \varphi|+|\rho|^{1 / 2}|\varphi \wedge \bar{\partial} \rho|\right\|_{\infty} .
$$

Note that the gradient of $u_{2}$ grows as the reciprocal of the distance to the boundary but has components in the complex directions parallel to the boundary which are of size $C|\rho|^{-1 / 2}$. This implies that $\left|u\left(z_{1}\right)-u\left(z_{2}\right)\right| \leq C$ whenever $z_{2} \in B\left(z_{1}\right)$, which clearly implies that $u \in B M O_{p}$ for any $p \geq 1$.

The proof of Lemma 3.1 is postponed until $\S 6$.

As described earlier, this lemma is sufficient to prove Theorem 2.4. For later use, remember that what will actually be proven is that $u=u_{1}+u_{2}$ where $u_{1} \in L^{\infty}$ and the gradient of $u_{2}$ behaves like the derivative of a Bloch function. In particular $f \in B D A_{p}$ implies that $f=f_{1}+g+h$, where $f_{1}$ satisfies condition (c) of Theorem $\mathrm{B}, h$ is analytic, and $\left|g\left(z_{1}\right)-g\left(z_{2}\right)\right| \leq C \beta\left(z_{1}, z_{2}\right)$ for all $z_{1}$ and $z_{2}$ in $D$. If $f \in B M O_{p}$ then the same is true with $h \equiv 0$ (because then $h \in \mathscr{B}$ and it can be absorbed in the $g$ ).

We now show that the Bergman projection $P$ is a bounded operator from $B M O_{p}$ to $\mathscr{B}$ that moreover takes $V M O_{p}$ to $\mathscr{B}_{0}$.

3.2. Proposition. Let $p \geq 1$. If $f \in B M O_{p}$ then $P f \in \mathscr{B}$ (the Bloch space) and so $(I-P) f \in B M O_{p}$. If $f \in V M O_{p}$ then $P f \in \mathscr{B}_{0}$ and $(I-P) f \in V M O_{p}$. Proof. If $f \in B M O_{p}$ then $f=f_{1}+g$ where $f_{1}$ satisfies condition (c) in Theorem $\mathrm{B}$, that is,

$$
\sup _{z \in D}\left(\frac{1}{|B(z)|} \int_{B(z)}\left|f_{1}\right|^{p} d V\right)^{1 / p}<\infty,
$$

while $g$ satisfies a Lipschitz condition:

$$
\left|g\left(z_{1}\right)-g\left(z_{2}\right)\right| \leq C \beta\left(z_{1}, z_{2}\right) .
$$

We first show that $P g \in \mathscr{B}$. By Definition 2.2, for this it suffices to show that $\rho(z) \partial(P g)(z)$ is bounded. Using Fefferman's formula [Fe] for the Bergman kernel $K(z, w)$, it follows that

$$
\left|\partial K(z, w) / \partial z_{k}\right| \leq C|K(z, w)||\Psi(z, w)|^{-1} \leq C \Psi(z, w)^{-n-2},
$$

where $\Psi(z, w)$ is defined in $\S 5$. (It is essentially the same as $X(z, w)$ in $[\mathrm{Fe}]$ and $\widehat{\boldsymbol{\Phi}}(z, w)$ in [R].) Now, using estimates given in [Li1, Theorem 4 and Corollary 11], we obtain $\beta(z, w) \leq C_{\varepsilon}|\rho(z)|^{1-\varepsilon}|\rho(w)|^{-\varepsilon}|K(z, w)|^{-2 \varepsilon /(n+1)}$ for 
sufficiently small $\varepsilon$. Thus

$$
\begin{aligned}
\left|\frac{\partial(P g)}{\partial z_{k}}\right| & =\left|\int_{D} g(w) \frac{\partial K(z, w)}{\partial z_{k}} d V(w)\right| \\
& =\left|\int_{D}(g(w)-g(z)) \frac{\partial K(z, w)}{\partial z_{k}} d V(w)\right| \\
& \leq C \int_{D} \beta(z, w)|K(z, w)||\Psi(z, w)|^{-1} d V(w) \\
& \leq C_{\varepsilon} \int_{D}|\rho(z) \rho(w)|^{-\varepsilon}|\Psi(z, w)|^{-n-2+2 \varepsilon} d V(w) \\
& \leq C C_{\varepsilon} /|\rho(z)|,
\end{aligned}
$$

where the last inequality comes from $[R$, Lemma 7.3, p. 310]. This is what was required for $g$.

It is clear from the condition on $f_{1}$ and Hölder's inequality that the function

$$
\frac{1}{|B(z)|} \int_{B(z)}\left|f_{1}\right| d V
$$

is bounded. For each $z \in D$ let $E(z)$ be a polydisk centered at $z$ with radii $c|\rho(z)|$ in the complex directions normal to the boundary and $c \sqrt{|\rho(z)|}$ in the complex directions parallel to the boundary. Choose $c$ so that $E(z) \subset$ $B(z)$ for all $z$ and recall that $\inf _{w \in B(z)}|E(w)| \sim|B(z)|$. Now $K(z, w)=$ $(1 /|E(w)|) \int_{E(w)} K(z, \zeta) d V(\zeta)$, so that by Fubini's theorem

$$
\begin{aligned}
P f_{1}(z) & =\int_{D} f_{1}(w) K(z, w) d V(w) \\
& =\int_{D}\left[\int_{D} f_{1}(w) \frac{\chi_{E(w)}(\zeta)}{|E(w)|} d V(w)\right] K(z, \zeta) d V(\zeta) .
\end{aligned}
$$

Since $\chi_{E(w)}(\zeta) \leq \chi_{B(w)}(\zeta)=\chi_{B(\zeta)}(w)$, it is clear that the expression in brackets above is a bounded function. Thus $P f_{1}$, being the projection of a bounded function, belongs to $\mathscr{B}$. (See $[\mathrm{C}]$.)

The $V M O_{p}$ case is entirely similar.

Combining Theorem B, Theorem 2.4 and Proposition 3.2 gives us the following:

3.3. Theorem. Let $f \in L^{1}$ and $p>1$. Then $H_{f}$ is bounded from $A^{p}$ to $L^{p}$ if and only if $(I-P) f \in B M O_{p}$.

Proof. If $(I-P) f \in B M O_{p}$ then $P f \in L^{1}$ and $H_{P f}=0$. Thus $H_{f}=H_{(I-P) f}$ is bounded. Conversely, if $H_{f}$ is bounded then, by Theorem $\mathrm{B}, f$ belongs to $B D A_{p}$ and, by Theorem 2.4, $f \in B M O_{p}+\mathscr{H}$. The holomorphic part will lie in $L^{1}$, and $I-P$ applied to it will give 0 . The conclusion now follows by Proposition 3.2.

\section{BMO AND RELATED SPACES}

Perhaps as interesting as the main theorem are some of its consequences. In contrast to the classical $V M O$ on $\mathbb{R}$ or $\partial \mathbb{D}$, the balls $B(z)$ over which the oscillation is averaged do not vary in size and it is only at the boundary ("at 
infinity" so to speak) that the mean oscillation conditions are of consequence. Moreover, the best that can be said of the local behavior of a function in $B M O_{p}$ or $V M O_{p}$ is that it is locally in $L^{p}$. One consequence of this difference is the fact that the spaces $V M O_{p}$ and $B M O_{p}$ vary with $p$. Another consequence is that the analogy between $B M O$ and $L^{\infty}$ in the classical setting is replaced by an analogy between $B M O_{p}$ and $L_{p}^{\infty}$, where $L_{p}^{\infty}$ will be defined below. Moreover, the continuous functions no longer play the same rôle in the theory. Nevertheless the fact that $H_{f}$ is bounded on $L^{p}$ if and only if $f \in B M O_{p}+\mathscr{H}$ and compact if and only if $f \in V M O_{p}+\mathscr{H}$ suggests that $B M O$ and $V M O$ play similar rôles in both settings. This is reinforced by the decomposition and duality results to follow.

4.1. Definition. Let $0<p, q \leq \infty$ and let $f$ be a measurable function on $\mathbb{D}$. We say that $f$ belongs to $L_{q}^{p}$ if the function

$$
z \mapsto\left(\frac{1}{|B(z)|} \int_{B(z)}|f|^{q} d V\right)^{1 / q}
$$

belongs to $L^{p}$. If $q=\infty$ we naturally replace the above function with

$$
z \mapsto \underset{w \in B(z)}{\operatorname{ess} \sup }|f(w)| .
$$

We give $L_{q}^{p}$ the obvious "norm", and it is a Banach space when $p$ and $q$ are both at least 1 . In case $1 \leq p, q<\infty$ the dual of $L_{q}^{p}$ is $L_{q^{\prime}}^{p^{\prime}}$ with the pairing $\langle f, g\rangle=\int_{D} f \bar{g} d V$. In this notation, the condition on $f_{1}$ in part (c) of Theorem B is that $f_{1} \in L_{p}^{\infty}$.

The following result will be stated in the context of the unit disk $\mathbb{D}$ in $\mathbb{C}$. Afterwards, corresponding results in a strongly pseudoconvex domain in $\mathbb{C}^{n}$ will be discussed briefly.

Recall that $T$ is the integral operator on $L^{p}$ of $\mathbb{D}$ whose kernel is $T(z, w)=$ $[\pi(z-w)(1-\bar{w} z)]^{-1}$, and $P$ is the Bergman projection whose kernel is $K(z, w)$ $=[\pi(1-\bar{w} z)]^{-2}$.

4.2. Theorem. If $f \in B M O_{p}$ then there exist functions $f_{1} \in L_{p}^{\infty}, \varphi \in L^{\infty}$ and $\psi \in L^{\infty}$ such that

$$
f=f_{1}+T \varphi+P \psi .
$$

The functions can be found to satisfy estimates

$$
(1 / C)\|f\|_{B M O, p} \leq\left\|f_{1}\right\|_{L_{p}^{\infty}}+\|\varphi\|_{\infty}+\|\psi\|_{\infty} \leq C\|f\|_{B M O, p} .
$$

Proof. Let $f \in B M O_{p}$. Clearly $f \in B D A_{p}$, and so $f=f_{1}+f_{2}$ as in part (c) of Theorem B. We have seen that $(I-P) f_{2}=T(\varphi)$ with $\varphi(w)=\bar{\partial} f_{2}(w)\left(1-|w|^{2}\right)$, so $f=f_{1}+T \varphi+h$ with $h$ holomorphic and $\varphi$ bounded. Clearly $h$ is also in $B M O_{p}$, and so the results in [Ax2] show that $h \in \mathscr{B}$, whence (see [Ax1]) $h=P \psi$ with $\psi$ bounded as required. The construction in [Lu3] gives an estimate on the first and second term of the sum in (7). The results in [Ax1] give an estimate on the third. Combined, these give the right-hand inequality of (7). Given a function $f$ of the form (6), the boundedness of $T$ and $P$ from $L^{\infty}$ to $B M O_{p}$ gives the left-hand inequality.

In a strongly pseudoconvex domain the following formulation seems the best we can do for now. 
4.3. Theorem. If $f \in B M O_{p}$ then there exists functions $f_{1}, f_{2}$, and $\psi$ such that $f_{1} \in L_{p}^{\infty}, f_{2}$ satisfies the conditions of part (c) of Theorem $\mathbf{B}, \psi$ is bounded, and

$$
f=f_{1}+\int_{D} \bar{\partial} f_{2}(w) \wedge L(\cdot, w)+P \psi,
$$

where $L$ is the kernel for the solution of the $\bar{\partial}$-equation constructed in $\S 6$. The functions can be found to satisfy estimates

$$
\begin{aligned}
(1 / C)\|f\|_{B M O, p} & \leq\left\|f_{1}\right\|_{L_{p}^{\infty}}+\left\|\left|\rho \bar{\partial} f_{2}\right|+|\rho|^{1 / 2}\left|\bar{\partial} f_{2} \wedge \bar{\partial} \rho\right|\right\|_{\infty}+\|\psi\|_{\infty} \\
& \leq C\|f\|_{B M O, p} .
\end{aligned}
$$

Proof. The proof goes just like the proof of Theorem 4.2 except that Coupet's work [C] is cited instead of Axler's and Li's [Li2] instead of Luecking's

In the statement of Theorem 4.3 any kernel that solves the $\bar{\partial}$-equation and satisfies the estimates of Lemma 6.4 may replace $L$.

We now define a version, suitable for our setting, of atomic $H^{1}$. In a sense, this goes to the opposite extreme from both the standard atomic theory and the "local" atomic theory of D. Goldberg ([Go]) in that the support of our atoms is not allowed to vary in size.

4.4. Definition. Let $q>1$. A $q$-atom is a measurable function $a(z)$, locally in $L_{q}$, such that

(a) $a$ is supported by some ball $B\left(z_{a}\right)$ ( $r$ is fixed),

(b) $\int_{D} a d V=0$,

(c) $\left(\left(1 /\left|B\left(z_{a}\right)\right|\right) \int_{B\left(z_{a}\right)}|a|^{q} d V\right)^{1 / q}=\left|B\left(z_{a}\right)\right|^{-1}$.

The space $H_{q}^{1}$ is defined to consist of all measurable functions $f$ which can be represented as an $l^{1}$ sum of atoms: $f=\sum_{j} \lambda_{j} a_{j}$ with $\sum_{j}\left|\lambda_{j}\right|<\infty$. The norm of a function in $H_{q}^{1}$ is defined to be the infimum of the sums $\sum\left|\lambda_{j}\right|$ over all such representations of $f$.

It is easy to verify that there is a constant $C=C(q, r)$ such that a $q$ atom has $L_{q}^{1}$ norm greater than $1 / C$ and less than $C$ so that $H_{q}^{1} \subset L_{q}^{1}$ and $\|f\|_{L_{q}^{1}} \leq C\|f\|_{H_{q}^{1}}$.

4.5. Proposition. Let $1<p, q<\infty$ with $\frac{1}{p}+\frac{1}{q}=1$. Then $V M O_{p}^{*}=H_{q}^{1}$ and $\left(H_{q}^{1}\right)^{*}=B M O_{p}$.

The pairing is the sesquilinear one $\langle f, g\rangle=\int_{D} f \bar{g} d V$ and is to be taken in the following sense: Functions with compact support in $D$ are dense in $H_{q}^{1}$ and the integral makes sense when $f$ is such a function. For arbitrary functions in $H_{q}^{1}$ the integral is obtained as a limit of the compactly supported case.

Proof. Let $g=\sum \lambda_{j} a_{j} \in H_{q}^{1}$, where each $a_{j}$ is a $q$-atom supported in a ball $B_{j}$ and chosen so that $\sum\left|\lambda_{j}\right| \leq(1+\varepsilon)\|g\|_{H_{q}}$. For a function $f \in V M O_{p}$ choose constants $c_{j}$ so that

$$
\left(\frac{1}{\left|B_{j}\right|} \int_{B_{j}}\left|f-c_{j}\right|^{p} d V\right)^{1 / p} \leq(1+\varepsilon)\|f\|_{B M O, p} .
$$


Then we have

$$
\begin{aligned}
|\langle f, g\rangle| & =\left|\sum_{j} \lambda_{j} \int f \bar{a}_{j} d V\right|=\left|\sum_{j} \lambda_{j} \int_{B_{j}}\left(f-c_{j}\right) \bar{a}_{j} d V\right| \\
& \leq \sum_{j}\left|\lambda_{j}\right|\left(\int_{B_{j}}\left|f-c_{j}\right|^{p} d V\right)^{1 / p}\left(\int_{B_{j}}\left|a_{j}\right|^{q} d V\right)^{1 / q} \\
& \leq \sum_{j}\left|\lambda_{j}\right|(1+\varepsilon)\|f\|_{B M O, p} \leq(1+\varepsilon)^{2}\|g\|_{H_{q}^{1}}\|f\|_{B M O, p} .
\end{aligned}
$$

Thus $g$ defines a continuous linear functional on $V M O_{p}$ whose norm is dominated by its $H_{q}^{1}$ norm. If $g$ happens to be a $q$-atom, let $\|g\|$ denote its $H_{q}^{1}$ norm and let $\|g\|_{*}$ denote its norm as a linear functional. Then we have $\|g\|_{*} \leq\|g\| \leq C$ for $C=C(q, r)$. On the other hand $\|g\|_{*}=\sup \left|\int_{B} f \bar{g} d V\right|$, where $B$ is the ball supporting $g$ in the definition of atom and the supremum is taken over $f \in V M O_{p}$ with norm 1 . Select any function $\psi$ in $L^{p}(B, d V /|B|)$ with distance to the constants equal to 1 . Extend $\psi$ to a function $f$ in $V M O_{p}$ by defining it to be constant outside $B$ using the constant $c$ which achieves the distance: $\int_{B}|\psi-c|^{p} d V /|B|=1$. Then $f$ is a norm 1 element of $V M O_{p}$ and the supremum of $\int f \bar{g} d V$ over all such $f$ is $|B|\left(\int_{B}|g|^{q} d V /|B|\right)^{1 / q}=1$. Thus $1 \leq\|g\|_{*} \leq\|g\| \leq C$. Moreover, the definitions show that the supremum over all $q$-atoms $g$ of $\left|\int f \bar{g} d V\right|$ is equal to $\|f\|_{B M O, p}$. Thus the $q$-atoms form a bounded norming subset of the dual of $V M O_{p}$, and now standard functional analysis arguments (see [Bo]) show that every element of the dual is an $l^{1}$ sum of $q$-atoms with the appropriate equivalence of norms.

Since different choices for the radius $r$ of $B(z)$ in the definition of $V M O_{p}$ give the same space with equivalent norms, we see that the same is true of $H_{q}^{1}$. Thus any function $f$ in $L^{1}(D, d V)$ which is supported on a ball $B\left(z, r^{\prime}\right)$ of any size and satisfies $\int f d V=0$ will belong to $H_{q}^{1}$.

The argument at the beginning of the proof also works to show that $B M O_{p}$ embeds in the dual of $H_{q}^{1}$ with the dual norm dominated by the $B M O_{p}$ norm. If now $\Lambda$ is a linear functional on $H_{q}^{1}$, then for each ball $B=B(z, r), \Lambda$ is defined on the subset of $L^{q}(B, d V /|B|)$ of functions of mean 0 . The functions in this space having norm $1 /|B|$ are $q$-atoms. Thus $\Lambda$ is associated to a family of functions $\left\{g_{B}\right\}$ by $\Lambda(f)=\int_{B} f \bar{g}_{B} d V /|B|$, each $g_{B}$ lying in $L^{p}(B, d V /|B|)$ and having distance from the constants at most $\|\Lambda\|$. If two such balls overlap, it is easy to show that the corresponding functions differ by a constant on the overlap. (Their difference annihilates all functions with mean 0 .) The problem now is to construct a globally defined function $g$ whose value on any given ball $B=B(z, r)$ differs from the corresponding $g_{B}$ by only a constant. For then

$$
\begin{aligned}
\|g\|_{B M O, p}^{p} & =\sup _{z} \inf _{c} \frac{1}{|B(z, r)|} \int_{B(z, r)}|g-c|^{p} d V \\
& =\sup _{z} \inf _{c} \frac{1}{|B(z, r)|} \int_{B(z, r)}\left|g_{B}-c\right|^{p} d V \leq\|\Lambda\|^{p} .
\end{aligned}
$$

To this end fix a point $z_{0}$ in $D$. Let $r<r_{1}<r_{2}<\cdots$ with $r_{k} \rightarrow \infty$. Then inductively obtain $g_{1}, g_{2}, \ldots$ such that for every $k \geq 1, g_{k}$ represents $\Lambda$ on 
$B\left(z_{0}, r_{k}\right)$. Adjust each $g_{k}$ by a constant if necessary so that $g_{k}=g_{k-1}$ on $B\left(z_{0}, r_{k-1}\right)$. Then $g$ is well defined by $g(z)=g_{k}(z)$ on $B\left(z_{0}, r_{k}\right)$ and agrees up to a constant with $g_{B}$ on every $B=B(z, r)$. Thus, for every $q$-atom $f$, $\Lambda(f)=\int f \bar{g} d V$.

We now define a space $V_{q}^{\infty}$ related to $L_{q}^{\infty}$ in the same way that $V M O$ is related to $B M O$.

4.6. Definition. If $f \in L_{q}^{\infty}$ we say that $f \in V_{q}^{\infty}$ if $\left((1 /|B(z)|) \int_{B(z)}|f|^{q} d V\right)^{1 / q}$ $\rightarrow 0$ as $z \rightarrow \partial D$.

Soon we will need the following small lemma, which is to $V M O_{p}$ as Theorem 4.2 is to $B M O_{p}$.

4.7. Lemma. Every $f \in V M O_{p}$ can be written

$$
f=f_{1}+T \varphi+P \psi
$$

where $f_{1} \in V_{p}^{\infty}, \varphi \in C_{0}$, and $\psi \in C_{0}$. The estimates of Theorem 4.2 (inequalities (7)) hold for $f, f_{1}, \varphi$, and $\psi$.

Proof. The proof is just like the proof of Theorem 4.2 but is based on the corresponding compactness criteria in [Lu3] instead of the boundedness criteria. Naturally, there is an analogous result for strongly pseudoconvex domains along the lines of Theorem 4.3.

Keeping the convention that $1 / p+1 / q=1$, we turn to a closer examination of the space $H_{q}^{1}$. It arises as the predual of $B M O_{p}$, which itself arises as the set of symbols $f$ for which $H_{f}$ is bounded. Or rather $B M O_{p}+\mathscr{H}$ does. The estimates of Theorem B and Lemma 3.1 indicate that for a given function $f$,

$$
\|f\|_{B D A, p} \leq \inf _{h \in \mathscr{H}}\|f-h\|_{B M O, p} \leq C\|f\|_{B D A, p} .
$$

Since $B M O_{p} \cap \mathscr{H}=\mathscr{B}$ (respectively, $V M O_{p} \cap \mathscr{H}=\mathscr{B}_{0}$ ), the $B D A_{p}$ norm of a function in $B M O_{p}$ (respectively, $V M O_{p}$ ) coincides with the norm in $B M O_{p} / \mathscr{B}$ (respectively, $V M O_{p} / \mathscr{B}_{0}$ ). The predual of $B M O_{p} / \mathscr{B}$ (respectively, the dual of $\left.V M O_{p} / \mathscr{B}_{0}\right)$ is the subset of functions in $H_{q}^{1}$ that annihilate analytic functions, that is, $\mathscr{B}_{0} \stackrel{\text { def }}{=}\left\{f \in H_{q}^{1}: \int f \bar{g} d V=0\right.$ for all $\left.g \in \mathscr{B}_{0}\right\}$. Now the $B M O_{p} / \mathscr{B}$ norm of a function $f$ is equivalent to the norm of the operator $H_{f}$ from $A^{p}$ to $L^{p}$, and this in turn is equivalent to

$$
\sup \left\langle H_{f} g_{1}, g_{2}\right\rangle=\sup \int f g_{1} \bar{g}_{2} d V
$$

where $g_{1}$ varies through the unit ball of $A^{p}$ and $g_{2}$ varies through the unit ball of $\left(A^{p}\right)^{\perp} \subset L^{q}=L_{q}^{q}$. Since $A^{p} \subset L_{\infty}^{p}$ it follows that $\bar{g}_{1} g_{2} \in L_{q}^{q} L_{\infty}^{p} \subset L_{q}^{1}$. Moreover the finiteness of $(10)$ shows that $\bar{g}_{1} g_{2} \in H_{q}^{1}$, and it clearly annihilates analytic functions. Applying the same functional analysis arguments as before, we conclude that

$$
\begin{aligned}
& \left(V M O_{p} / \mathscr{B}_{0}\right)^{*} \\
& =\mathscr{B}_{0}^{\perp}=\left\{\sum_{j} \bar{h}_{j} g_{j}: h_{j} \in A^{p}, g_{j} \in\left(A^{p}\right)^{\perp}, \sum\left\|h_{j}\right\|_{p}\left\|g_{j}\right\|_{q}<\infty\right\} .
\end{aligned}
$$


Moreover, this is also the predual of $B M O_{p} / \mathscr{B}$. This gives us half of the first equivalence in the following theorem. Let $A_{0}^{p}$ denote the class of functions $f$ in $A^{p}$ with $\int_{D} f d V=0$.

4.8. Theorem. The following are equivalent for a function $f$ in $L_{q}^{1}$ :

(a) $f \in H_{q}^{1}$, that is, $f$ is an $l^{1}$ sum of q-atoms.

(b) $f=h+\sum_{j} \bar{h}_{j} g_{j}$ with $h \in A_{0}^{1}$, each $h_{j} \in A^{p}$, each $g_{j} \in\left(A^{p}\right)^{\perp}$ and $\sum\left\|h_{j}\right\|_{p}\left\|q_{j}\right\|_{q}<\infty$.

(c) In case $n=1$ and $D=\mathbb{D}, P f \in L^{1}$ and $T^{*} f \in L^{1}$.

The decomposition in (b) can be associated with a norm, namely the infimum of $\|h\|_{1}+\sum_{j}\left\|h_{j}\right\|_{p}\left\|g_{j}\right\|_{q}$ over all such decompositions. This norm is equivalent to the atomic norm. Similarly the sum $\|f\|_{L_{q}^{1}}+\|P f\|_{1}+\left\|T^{*} f\right\|_{1}$ is an equivalent norm. The operator $T^{*}$ is the formal adjoint of $T$, that is, the operator with kernel $T^{*}(z, w)=\overline{T(w, z)}$.

Proof. We have seen that $P$ is bounded from $V M O_{p}$ to $\mathscr{B}_{0}$, and we will see (in $\S 5$ ) that $P$ is also bounded from $H_{q}^{1}$ to $L^{1}$. Thus if $f \in H_{q}^{1}$ then we have $f=P f+(I-P) f$. The second term belongs to $\mathscr{B}_{0}^{\perp}$ and the first to $A_{0}^{1}$. Then (b) follows from (a) by the preceding discussion. Conversely, let $f$ have the form in (b). We need only show that $A_{0}^{1} \subset H_{q}^{1}$ to conclude that $f \in H_{q}^{1}$. But given $h \in A_{0}^{1}$ and $g \in C(\bar{D})$ we have

$$
\int_{D} h \bar{g} d V=\int_{D} P h \bar{g} d V=\int_{D} h \overline{P g} d v .
$$

It is known that $A_{0}^{1}$ is the dual of $\mathscr{B}_{0}$, and so

$$
\left|\int_{D} h \overline{P g} d V\right| \leq C\|h\|_{1}\|P g\|_{\mathscr{B}} \leq C\|h\|_{1}\|g\|_{B M O, p} .
$$

We conclude that as a linear functional on $V M O_{p}, h$ agrees with an element of $H_{q}^{1}$. It is easy to see that this means they are the same function.

Now let $n=1$ and assume (a). We have already mentioned that $P f \in L^{1}$ when $f \in H_{q}^{1}$, and it is similarly easy to show that $T^{*}$ is bounded from atoms to $L^{1}$. This gives the implication $(\mathrm{a}) \Rightarrow(\mathrm{c})$.

Now suppose that $f$ is a function in $L_{q}^{1}$ such that $P f$ and $T^{*} f$ are in $L^{1}$. To show that $f$ is in $H_{q}^{1}$ it suffices to show that it defines a bounded linear functional on $V M O_{p}$. To this end let $g \in V M O_{p}$ with $g=g_{1}+T \varphi+P \psi$, where $g_{1}, \varphi$ and $\psi$ have compact support, and observe that

$$
\begin{aligned}
\langle f, g\rangle & =\left\langle f, g_{1}\right\rangle+\langle f, T \varphi\rangle+\langle f, P \psi\rangle \\
& =\left\langle f, g_{1}\right\rangle+\left\langle T^{*} f, \varphi\right\rangle+\langle P f, \psi\rangle .
\end{aligned}
$$

It follows easily that

$$
\begin{aligned}
|\langle f, g\rangle| & \leq\|f\|_{L_{q}^{1}}\left\|g_{1}\right\|_{L_{p}^{\infty}}+\left\|T^{*} f\right\|_{1}\|\varphi\|_{\infty}+\|P f\|_{1}\|\psi\|_{\infty} \\
& \leq\left(\|f\|_{L_{q}^{1}}+\left\|T^{*} f\right\|_{1}+\|P f\|_{1}\right)\|g\|_{B M O, p} .
\end{aligned}
$$

Since functions with compact support are dense in $V_{p}^{\infty}$ as well as $C_{0}$, this defines $f$ as a bounded linear functional on a dense subset of $V M O_{p}$, and so (c) $\Rightarrow(\mathrm{a})$. 
Remark. It is a little more complicated to state a strongly pseudoconvex domain version of part (c) of Theorem 4.8, but here is an indication of how to go about it for the interested reader: Use the sup norm of the expression

$$
\sup _{z \in D}\left(|\rho(z) \varphi(z)|+|\rho(z)|^{1 / 2}|\bar{\partial} \rho(z) \wedge \varphi(z)|\right)
$$

to define an $L^{\infty}$-type space of forms. Its predual will be an appropriate $L^{1}$-type space of forms. In place of $T$ use the operator $\varphi \mapsto \int \varphi \wedge L(\cdot, w)$ from $\S 6$ (call it simply $L$ ), and then in place of (c) will be a similar statement involving $L^{*}$ and that $L^{1}$-type space.

\section{BOUNDEDNESS OF HANKEL OPERATORS IN $L^{1}$ NORM}

Since $A_{0}^{1}$ is contained in $H_{q}^{1}$ and the operators $T, T^{*}$, and $P$ are bounded on $H_{q}^{1}$ it makes sense to consider the boundedness of various operators on $A^{1}$ defined using these operators. Here we investigate the boundedness of Hankel operators from $A^{1}$ to $L^{1}$. The crucial problem, which makes this case different from the rest, is this: multiplying by a bounded function is not in general bounded from $A^{1}$ to $H_{q}^{1}$ for any $q$. It is bounded from $A^{1}$ to $L^{1}$, but then neither $P$ nor $T$ is bounded on $L^{1}$. We do not at present have a complete characterization of the Hankel operators which are bounded in $L^{1}$ norm. We present here one necessary condition and one sufficient condition. We then show that for the important special case of the conjugate of an analytic function, the sufficient condition is also necessary. Finally, we present an example to show that, in general, the sufficient condition is not necessary (and a simple argument to show that the necessary condition is not sufficient).

The necessary condition we obtain is gotten by following the proof of the corresponding result for $A^{p}$ with $p>1$, but refraining from continuing to the last step (d) $\Rightarrow(a)$, which will not work.

5.1. Theorem. Let $f$ be a measurable function in $L^{1}$ and let $H_{f}$ be defined on bounded analytic functions. If $H_{f}$ extends to a bounded operator from $A^{1}$ to $L^{1}$ then the following equivalent conditions hold.

(a) For some (respectively, every) $r>0$,

$$
F(z) \stackrel{\text { def }}{=} \inf _{h \in \mathscr{C}} \frac{1}{|B(z, r)|} \int_{B(z, r)}|f-h| d V
$$

is bounded on $d$.

(b) $f=f_{1}+f_{2}$, where

$$
G(z) \stackrel{\text { def }}{=} \frac{1}{|B(z, r)|} \int_{B(z, r)}\left|f_{1}\right| d V
$$

is bounded on $D$ for some (respectively, every) $r>0$ and

$$
|\rho(z)|\left|\bar{\partial} f_{2}(z)\right|+|\rho(z)|^{1 / 2}\left|\bar{\partial} f_{2}(z) \wedge \partial \rho(z)\right|
$$

is bounded on $D$.

(c) $f \in B M O_{1}+\mathscr{H}$.

In particular, if $f$ is the conjugate of an analytic function $f=\bar{h}$ then it follows from (a), (b), or (c) that $h$ is in $\mathscr{B}$. The proof of Theorem 5.1 is 
omitted, as it is identical to the proofs in [Lu3] and [Li2]. (That (c) implies (a) is clear, so the conditions are indeed equivalent.)

To obtain a sufficient condition we can try to find additional conditions on $f_{1}$ and $f_{2}$ which will make both $H_{f_{1}}$ and $H_{f_{2}}$ bounded, and then impose conditions on $f$ that will produce these. Since the necessary condition on $f_{1}$ already implies $M_{f_{1}}$ is bounded from $A^{1}$ to $L^{1}$, the boundedness of $P M_{f_{1}}$ will imply the boundedness of $H_{f_{1}}$. A sufficient condition for the boundedness of $P M_{f_{1}}$ has already been obtained by $\mathrm{K}$. Zhu for the ball, and this leads to the sufficient condition we consider in the next theorem.

5.2. Theorem. Let $f$ be a measurable, locally integrable function on D. Suppose that the following function is bounded:

$$
z \mapsto \log \frac{1}{|\rho(z)|} \inf _{h \in \mathscr{R}} \frac{1}{|B(z)|} \int_{B(z)}|f-h| d V .
$$

Then there exists a decomposition $f=f_{1}+f_{2}$, where

$$
\log \frac{1}{|\rho(z)|} \frac{1}{|B(z, r)|} \int_{B(z, r)}\left|f_{1}\right| d V
$$

is bounded on $D$ for some (respectively, all) $r>0$ and

$$
\log \frac{1}{|\rho(z)|}\left[|\rho(z)|\left|\bar{\partial} f_{2}(z)\right|+|\rho(z)|^{1 / 2}\left|\bar{\partial} f_{2}(z) \wedge \partial \rho(z)\right|\right]
$$

is bounded on $D$. Moreover, this decomposition implies that $H_{f}$ extends to a bounded operator from $A^{1}$ to $L^{1}$.

Proof. The methods of [Lu3] and [Li2] show that the boundedness of (12) implies the decomposition in the same way that (b) implies (c) in Theorem B and in the previous theorem.

We need to establish a series of lemmas. The difficulty (at least for the general strongly pseudoconvex domain) is that we do not have an explicit integral formula for $H_{f_{2}} g(z)$. This makes us follow a somewhat convoluted path.

We begin with a construction from [Li2] of a kernel $L(z, w)$ which will solve the $\bar{\partial}$-equation with good estimates. Recall that $\rho$ is the strictly plurisubharmonic defining function for $D$. We use $\rho_{j}(z)$ for the derivative $\partial \rho(z) / \partial z_{j}$ and $\rho_{j k}$ for the corresponding second derivative, $1 \leq j, k \leq n$. Let $F(z, w)$ denote the Levi polynomial for $D$. That is,

$$
F(z, w)=\sum_{j=1}^{n} \rho_{j}(w)\left(w_{j}-z_{j}\right)-\frac{1}{2} \sum_{j=1}^{n} \sum_{k=1}^{\infty} \rho_{j k}(w)\left(w_{j}-z_{j}\right)\left(w_{k}-z_{k}\right) .
$$

It is well known that there exist constants $\delta$ and $c$ such that $F$ satisfies

$$
\operatorname{Re} F(z, w)+|\rho(w)| \geq \frac{|\rho(z)|+|\rho(w)|}{2}+c|z-w|^{2}
$$

for all $z, w \in \bar{D}$ with $|z-w|<\delta$. The following lemma is from [F].

5.3. Lemma. Let $\rho$ and $\delta$ be as above. There exist functions $h_{j}(z, w), 1 \leq$ $j \leq n$, and $\Psi(z, w)$ in $C^{\infty}(\bar{D} \times \bar{D})$ and a constant $C>0$ with the following properties.

(a) For each fixed $w \in \bar{D}, h_{j}(z, w)$ and $\Psi(\cdot, w)$ are holomorphic on $\bar{D}$. 
(b) $\Psi(z, w)=|\rho(w)|+\sum_{j=1}^{n} h_{j}(z, w)\left(w_{j}-z_{j}\right)$.

(c) $|\Psi(z, w)| \geq 1 / C$ when $|z-w|>\delta / 2$, and otherwise $\Psi(z, w)=$ $g(z, w)(F(z, w)+|\rho(w)|)$, where $g$ is smooth and nonvanishing on $\bar{D} \times \bar{D}$.

(d) $h_{j}(w, w)=\rho_{j}(w)-\rho(w) g_{j}(w)$, where $g_{j}(w)=\partial g(z, w) /\left.\partial w_{j}\right|_{z=w}$.

Now let

$$
\begin{gathered}
s(z, w)=\sum_{j=1}^{n}\left(\bar{w}_{j}-\bar{z}_{j}\right) d w_{j}, \quad h(z, w)=\sum_{j=1}^{n} h_{j}(z, w) d w_{j}, \\
q(z, w)=\frac{\bar{\partial}_{w} h(z, w)}{\rho(w)}-\frac{\bar{\partial} \rho(w) \wedge h(z, w)}{\rho(w)^{2}},
\end{gathered}
$$

and finally for any $N \geq 1$ let

$$
\begin{aligned}
& L^{(N)}(z, w) \\
& \quad=C_{n, N} \sum_{k=0}^{n-1} \dot{a_{k}}\left(\frac{\rho(w)}{\Psi(z, w)}\right)^{k+N} \frac{s(z, w) \wedge q(z, w)^{k} \wedge\left(\bar{\partial}_{w} s(z, w)\right)^{n-k-1}}{|z-w|^{2 n-2 k}}
\end{aligned}
$$

where $a_{k}$ and $C_{n, N}$ are constants whose exact value need not concern us. This formula and the lemma to follow may be found in [Li2] and ultimately in [BA, p. 102, equation (20)].

5.4. Lemma. Let $N \geq 1, f \in C^{1} \cap L^{1}$ on $D$ and $|\rho \bar{\partial} f|+|\rho|^{1 / 2}|\bar{\partial} \rho \wedge \bar{\partial} f| \in L^{1}$. Then, for appropriate choices of $C_{n, N}$ and $a_{k}$,

$$
f(z)=\int_{D} \bar{\partial} f(w) \wedge L^{(N)}(z, w)+a_{n} \int_{D} f(w)\left(\frac{\rho(w)}{\Psi(z, w)}\right)^{n+N} \wedge q(z, w)^{n},
$$

where $a_{n}$ is another constant whose value will not concern us. Moreover, if $\varphi$ is a $\bar{\partial}$-closed $(0,1)$-form with $|\rho \varphi|+|\rho|^{1 / 2}|\varphi \wedge \bar{\partial} \rho| \in L^{1}$, then

$$
u(z)=\int_{D} \varphi(w) \wedge L^{(N)}(z, w)
$$

solves the $\bar{\partial}$-equation $\bar{\partial} u=\varphi$ in $D$.

Let $L_{k}(z, w)$ denote the $k$ th term of the function for $L^{(N)}$ (omitting the constants). Thus

$$
L_{k}(z, w)=\left(\frac{\rho(w)}{\Psi(z, w)}\right)^{k+N} \frac{s(z, w) \wedge q(z, w)^{k} \wedge\left(\bar{\partial}_{w} s(z, w)\right)^{n-k-1}}{|z-w|^{2 n-2 k}} .
$$

For the purposes of our proof, let us remark that

$$
\begin{aligned}
q(z, w)^{k} & =\frac{\left(\rho \bar{\partial}_{w} h\right)^{k}-k\left(\rho \bar{\partial}_{w} h\right)^{k-1} \wedge h \wedge \bar{\partial} \rho}{\rho^{2 k}} \\
& =\rho^{-k-1}\left(\bar{\partial}_{w} h\right)^{k-1} \wedge\left(\rho \bar{\partial}_{w} h-k h \wedge \bar{\partial} \rho\right) .
\end{aligned}
$$

This is easily established by induction using $\bar{\partial} \rho \wedge \bar{\partial} \rho=0$. Thus

$$
\begin{aligned}
L_{k}(z, w) & =\frac{\rho(w)^{N-1} s(z, w) \wedge\left[\bar{\partial}_{w} s(z, w)\right]^{n-k-1}}{\Psi(z, w)^{k+N}|z-w|^{2 n-2 k}} \\
& \wedge\left[\rho(w) q_{1}(z, w)+q_{2}(z, w) \wedge \bar{\partial} \rho(w)\right]
\end{aligned}
$$


where $q_{1}$ and $q_{2}$ are both smooth and bounded. When $k=0$ we have $q_{1}=1$ and $q_{2}=0$. Thus we have the following estimates if $\varphi$ satisfies the condition of Lemma 3.1, namely $|\rho \varphi|+|\rho|^{1 / 2}|\varphi \wedge \bar{\partial} \rho|$ is bounded:

$$
\begin{aligned}
& \left|\varphi(w) \wedge L_{0}(z, w)\right| \leq C \frac{\rho(w)^{N-1}}{|\Psi(z, w)|^{N}|z-w|^{2 n-1}}, \\
& \left|\varphi(w) \wedge L_{1}(z, w)\right| \leq C \frac{\rho(w)^{N-3 / 2}}{|\Psi(z, w)|^{1+N}|z-w|^{2 n-3}}, \\
& \left|\varphi(w) \wedge L_{k}(z, w)\right| \leq C \frac{\rho(w)^{N-3 / 2}}{|\Psi(z, w)|^{3 / 2+N}|z-w|^{2 n-4}} \quad \text { if } k \geq 2,
\end{aligned}
$$

where the constant $C$ is a multiple of the $L^{\infty}$ norm of $|\rho \varphi|+|\rho|^{1 / 2}|\varphi \wedge \bar{\partial} \rho|$. The last inequality uses the fact that $|\Psi(z, w)| \geq c|z-w|^{2}$.

Now let $K^{(N)}(z, w)$ be the kernel in the second integral of the formula for $f(z)$ in Lemma 5.4 , that is,

$$
K^{(N)}(z, w) d V(w)=\left(\frac{|\rho(w)|}{\Psi(z, w)}\right)^{n+N} q(z, w)^{n},
$$

where $q$ and $\Psi$ are as in Lemma 5.3. Let $H(z, w)=K^{(1)}(z, w)$. Let $A(z, w)=\overline{H(w, z)}-H(z, w)$. Let $H$ and $A$ denote the integral operators with kernels $H(z, w)$ and $A(z, w)$, respectively.

5.5. Lemma. The Bergman projection $P$ may be written $P=H+P A$. Moreover, $|A(z, w)| \leq C|\Psi(z, w)|^{-n-1 / 2}$, and $\left|\nabla_{w} A(z, w)\right| \leq C|\Psi(z, w)|^{-n-3 / 2}$.

Proof. See [R, Chapter VII, §7].

5.6. Lemma. Let $t \geq 0$. The function $\Psi$ satisfies the following estimates for all $z \in D$ :

$$
\int_{D} \frac{|\rho(w)|^{t}}{|\Psi(z, w)|^{n+1+s+t}}+\frac{|\rho(w)|^{t}}{|\Psi(w, z)|^{n+1+s+t}} d V(w) \leq C \begin{cases}1 & \text { if } s<0, \\ |\log | \rho(z)|| & \text { if } s=0, \\ |\rho(z)|^{-s} & \text { if } s>0,\end{cases}
$$

and if $\varphi$ is a form satisfying the condition of Lemma 3.1 and if $N \geq 2$, then

$$
\int_{D}\left|\varphi(w) \wedge L^{(N)}(z, w)\right| d V(z) \leq C\left\||\rho \varphi|+|\rho|^{1 / 2}|\varphi \wedge \bar{\partial} \rho|\right\|_{\infty} .
$$

Proof. The first part is almost [R, Lemma 7.3, p. 310]. The proof there relies only on the fact that $|\Psi(z, w)| \geq c\left(|\rho(z)|+|\rho(w)|+|z-w|^{2}\right)$ on $\bar{D} \times \bar{D}$. Thus it is valid with the variables interchanged. That lemma is stated with $t=0$, but since $|\rho(w)| /|\Psi(z, w)| \leq C$ for all $z$ and $w$, it clearly implies our statement.

The last estimate can be obtained by first using the appropriate estimate from the list preceding Lemma 5.5, then using the same lower estimate on $|\Psi|$ as above, and making a standard change of variables $z \rightarrow t(z)$ with $\operatorname{Re} t_{1}(z)=$ $\rho(w)-\rho(z)$ as in [Li2] or [DH]. This leads to one of the integrals in [Li2, Lemma 3.2] or [DH, Lemma 2.2]. The estimates there (with $\alpha=0$ ) yield the estimate (16).

The main estimates we need are contained in the following lemma. 
5.7. Lemma. If $f \in C^{1}$ on $D$ with $\log (1 /|\rho|)\left(|\rho \bar{\partial} f|+|\rho|^{1 / 2}|\bar{\partial} f \wedge \bar{\partial} \rho|\right) \in L^{\infty}$ and $|f| \leq C(1+|\log (|\rho|)|)$, then the Hankel operator $H_{f}$ is bounded from $A^{1}$ to $L^{1}$.

Proof. Note that $H_{f}=(I-P) M_{f}=(I-H) M_{f}-P A M_{f}$. We estimate these two parts separately. Let $g$ be an analytic function, continuous on $\bar{D}$. Then by Lemma 5.4 with $N=1$ applied to $g$

$$
\begin{aligned}
(I-H)(f g)(z)= & \int(f(z)-f(w)) g(w) H(z, w) d V(w) \\
= & C \int(f(z)-f(w)) g(w) \\
& \times \frac{\left[\rho(w) \bar{\partial}_{w} h(z, w)-n \bar{\partial} \rho(w) \wedge h(z, w)\right] \wedge \bar{\partial}_{w} h(z, w)^{n-1}}{\Psi(z, w)^{n+1}}
\end{aligned}
$$

from equation (14). Continuing the above equations:

$$
\begin{aligned}
(I-H) & (f g)(z)=C f(z) \int g(w) \frac{\rho(w)\left(\bar{\partial}_{w} h(z, w)\right)^{n}}{\Psi(z, w)^{n+1}} \\
& -C \int f(w) g(w) \frac{\rho(w)\left(\bar{\partial}_{w} h(z, w)\right)^{n}}{\Psi(z, w)^{n+1}} \\
& -n C \int(f(z)-f(w)) g(w) \frac{\bar{\partial} \rho(w) \wedge h(z, w) \wedge\left(\bar{\partial}_{w} h(z, w)\right)^{n-1}}{\Psi(z, w)^{n+1}} \\
= & I_{1}(z)+I_{2}(z)+I_{3}(z) .
\end{aligned}
$$

For any $\alpha>1$ with $\alpha(n+1 / 2)<n+1$ one has from Lemma 5.6

$$
\int_{D}\left(\frac{|\rho(w)|^{1 / 2}}{|\Psi(z, w)|^{n+1}}\right)^{\alpha} d V(w)+\int_{D}\left(\frac{|\rho(w)|^{1 / 2}}{|\Psi(z, w)|^{n+1}}\right)^{\alpha} d V(z) \leq C .
$$

Note also that $|f(z)| \leq C(1+|\log (|\rho|)|)$ implies that $f \in L^{p}$ for all $p>0$ and $|\rho(z)|^{1 / 2} f(z) \in L^{\infty}$. The extension of Young's theorem [R, p. 358] together with (17) implies that the kernel

$$
\frac{|\rho(w)|^{1 / 2} \bar{\partial} h(z, w)^{n}}{\Psi(z, w)^{n+1}}
$$

is a bounded operator from $L^{1}$ to $L^{\alpha}$. This implies that $\left\|I_{1}\right\|_{1}+\left\|I_{2}\right\|_{1} \leq C\|g\|_{1}$.

To estimate $I_{3}$ we use integration by parts. Recall that $\Psi(z, w)=-\rho(w)+$ $\sum_{i=1}^{n} h_{i}(z, w)\left(w_{i}-z_{i}\right)$. By Stokes' theorem,

$$
\begin{aligned}
I_{3}(z)= & C\left[\int_{D}-\bar{\partial} f(w) g(w) \frac{\rho(w) \wedge h(z, w) \wedge \bar{\partial}_{w} h(z, w)^{n-1}}{\Psi(z, w)^{n+1}}\right. \\
& +\int_{D}(f(z)-f(w)) g(w) \frac{\rho(w) \wedge\left(\Psi(z, w) \bar{\partial}_{w} h(z, w)^{n}+H_{1}(z, w)\right)}{\Psi(z, w)^{n+2}} \\
& \left.+(n+1) \int_{D}(f(z)-f(w)) g(w) \frac{\rho(w) \bar{\partial} \rho(w) \wedge h(z, w) \wedge \bar{\partial}_{w} h(z, w)^{n-1}}{\Psi(z, w)^{n+2}}\right] \\
= & J_{1}(z)+J_{2}(z)+J_{3}(z),
\end{aligned}
$$

where $H_{1}$ is an $(n, n)$-form with $\left|H_{1}(z, w)\right| \leq C|z-w|$. By Lemma 5.6, one can easily estimate that

$$
\left\|J_{1}\right\|_{1} \leq C\|(\log |\rho|) \rho \bar{\partial} f\|_{\infty}\|g\|_{1} .
$$


Now observe that

$$
|\rho(w)|^{1 / 2} \frac{\left|\Psi(z, w)(\bar{\partial} h)^{n}+H_{1}(z, w)\right|}{|\Psi(z, w)|^{n+2}} \leq \frac{C}{|\Psi(z, w)|^{n+1}} .
$$

Then the same reasoning as in the estimates of $\left\|I_{1}\right\|_{1}$ and $\left\|I_{2}\right\|_{1}$ yields

$$
\left\|J_{2}\right\|_{1} \leq C\|g\|_{1} \text {. }
$$

By Lemma 5.4, it is easy to see that there are constants $C_{1}$ and $C_{2}$ such that

$$
\begin{aligned}
J_{3}(z)= & C_{1}\left[f(z) g(z)-\int_{D} f(w) g(w) K^{(2)}(z, w) d V(w)\right] \\
& +C_{2} \int_{D}(f(z)-f(w)) g(w) \frac{\rho(w)^{2} \wedge(\bar{\partial} h(z, w))^{n}}{\Psi(z, w)^{n+2}} \\
= & C_{1} \int_{D} L^{(2)}(z, w) \wedge \bar{\partial} f(w) g(w) \\
& +C_{2} \int_{D}(f(z)-f(w)) g(w) \frac{\rho(w)^{2} \wedge(\bar{\partial} h(z, w))^{n}}{\Psi(z, w)^{n+2}}
\end{aligned}
$$

By the same arguments as those used in the estimates of $\left\|I_{1}\right\|_{1}$ and $\left\|I_{2}\right\|_{1}$, we have that the $L^{1}$-norm of the second term in (18) is bounded by $C\|g\|_{1}$.

By the second part of Lemma 5.6

$$
\int_{D}\left|L^{(2)}(z, w) \wedge \bar{\partial} f(w)\right| d V(z) \leq C\left\||\rho \bar{\partial} f|+|\rho|^{1 / 2}|\bar{\partial} f \wedge \bar{\partial} \rho|\right\|_{\infty} .
$$

Thus

$$
\left\|J_{3}\right\|_{1} \leq C \int_{D} \int_{D}\left|L^{(2)}(z, w) \wedge \bar{\partial} f(w) g(w)\right| d V(z) d V(w) \leq C\|g\|_{1} .
$$

Thus we have shown that $\left\|(I-H) M_{f} g\right\|_{1} \leq C\|g\|_{1}$ for $g \in \mathscr{C} \cap C(\bar{D})$. Since such functions are dense in $A^{1}$ it follows that $(I-H) M_{f}$ is bounded from $A^{1}$ to $L^{1}$. We turn now to the boundedness of $P A M_{f}$.

Let $\left\{U_{i}: 1 \leq i \leq m\right\}$ be an open cover of $\bar{D}$ such that $\bar{U}_{m} \subset D, \partial D \subset$ $\bigcup_{i=1}^{m-1} U_{i}$, and such that in each $U_{k}$ we have $\left|\partial \rho / \partial z_{n_{k}}\right|>b>0$ for some $n_{k} \leq n$, where $b$ is a constant depending only on $\rho$. Let $\left\{\gamma_{j}\right\}$ be a smooth partition of unity subordinate to $\left\{U_{i}\right\}$. Then, by Stokes' theorem,

$$
\begin{aligned}
A(f g)(z)= & \sum_{k=1}^{m-1} \int_{D} A(z, w) \frac{\gamma_{k}(w)}{\partial \rho / \partial \bar{w}_{n_{k}}} f(w) g(w) \frac{\partial \rho}{\partial \bar{w}_{n_{k}}} d V(w) \\
& +\int_{D} \gamma_{m}(w) f(w) g(w) A(z, w) d V(w) \\
= & \sum_{k=1}^{m-1} \int_{D}\left[F_{k}(w) \frac{\partial A(z, w)}{\partial \bar{w}_{n_{k}}}+G_{k}(w) A(z, w)\right] \rho(w) f(w) g(w) d V(w) \\
& +\sum_{k=1}^{m-1} \int_{D} F_{k}(z, w) A(z, w) \rho(w) \frac{\partial f}{\partial \bar{w}_{n_{k}}} g(w) d V(w) \\
& +\int_{D} \gamma_{m}(w) f(w) g(w) A(z, w) d V(w),
\end{aligned}
$$


where $F_{k}=\gamma_{k} /\left(\partial \rho / \partial \bar{w}_{n_{k}}\right)$ and $G_{k}=\partial F_{k} / \partial \bar{w}_{n_{k}}$ are all smooth on $\bar{D}$. The assumptions imply that $\gamma_{m} f$ is bounded, so we get

$$
|A(f g)(z)| \leq C \int_{D} \frac{|\rho(w) f(w) g(w)|}{|\Psi(z, w)|^{n+3 / 2}}+\frac{|\rho(w) \bar{\partial} f(w) g(w)|+|g(w)|}{|\Psi(z, w)|^{n+1 / 2}} d V(w) .
$$

The hypotheses on $f$ imply that $|\rho|^{1 / 4} f$ and $\rho \bar{\partial} f$ are bounded. Since $\Psi$ and $\rho / \Psi$ are also bounded, we get

$$
|A(f g)(z)| \leq C \int_{D} \frac{|g(w)|}{|\Psi(z, w)|^{n+3 / 4}} d V(w) .
$$

For any $\alpha>1$ with $\alpha(n+3 / 4)<n+1$ we get from Lemma 5.6

$$
\int_{D}\left|\Psi(z, w)^{-n-3 / 4}\right|^{\alpha} d V(w)+\int_{D}\left|\Psi(z, w)^{-n-3 / 4}\right|^{\alpha} d V(z) \leq C .
$$

Then [R, p. 358] and the preceding inequalities imply that $\|A(f g)\|_{\alpha} \leq C\|g\|_{1}$. Since $P$ is bounded on $L^{\alpha}$ for any $\alpha>1$, we have $\|P A(f g)\|_{1} \leq C\|P A(f g)\|_{\alpha}$ $\leq C\|g\|_{1}$, and the proof of Lemma 5.7 is complete.

Proof (of Theorem 5.2). The only thing that prevents us from using Lemma 5.7 directly is the hypothesis that $|f(z)| \leq C(1+|\log | \rho(z)||)$ and the fact that the constants depended on this $C$. To get around this we write $f=f_{1}+f_{2}$ as in the hypothesis. Let $u(z)$ be the solution to $\bar{\partial} u=\bar{\partial} f_{2}$ guaranteed by Lemma 3.1, and observe two things: (1) Since $\bar{\partial} u=\bar{\partial} f_{2}, u$ satisfies the same condition as $f_{2}$. (2) Since $f_{2}$ satisfies (13), it satisfies the weaker condition

$$
\left|\rho(z) \bar{\partial} f_{2}(z)\right|+|\rho(z)|^{1 / 2}\left|\bar{\partial} f_{2}(z) \wedge \partial \rho(z)\right| \in L^{\infty},
$$

and this is sufficient by Lemma 3.1 to give $|u(z)-u(w)| \leq C(1+\beta(z, w))$ with the constant $C$ depending only on the $L^{\infty}$ norm of the above expression. This easily implies

$$
|u(z)| \leq C(1+|\log | \rho(z)||) .
$$

Thus $H_{u}$ is bounded by Lemma 5.7, and so $H_{f_{2}}$ is bounded since it equals $H_{u}$.

To finish, we examine $H_{f_{1}}$. Again the condition on $f_{1}$ implies a weaker condition:

$$
\frac{1}{|B(z, r)|} \int_{B(z, r)}\left|f_{1}\right| d V \in L^{\infty}
$$

This implies that $M_{f_{1}}$ is bounded from $A^{1}$ to $L^{1}$, and it remains to show that $P M_{f_{1}}$ is also bounded. Now $P M_{f_{1}} g(z)=\int_{D} K(z, w) f_{1}(w) g(w) d V(w)$ and $\int_{D}|K(z, w)| d V(w) \leq C(1+|\log | \rho(w)||)$, which implies that

$$
\left\|P M_{f_{1}} g\right\|_{1} \leq C \int_{D}(1+|\log | \rho||)\left|f_{1}\right||g| d V .
$$

Letting $f_{3}=(1+|\log | \rho||) f_{1}$ and observing that $\rho(z) \sim \rho(w)$ when $z, w \in$ $B(z, r)$, we conclude that $f_{3}$ also satisfies the condition (19). Thus $f_{3}$ is a multiplier from $A^{1}$ to $L^{1}$, and this means the right-hand side of $(20)$ is bounded by a multiple of the $L^{1}$ norm of $g$.

Our next result is to show the converse of Theorem 5.2 when the symbol $f$ is the conjugate of an analytic function. 
Recall the definition of $q$-atom from $\S 4$. These are functions $a$ which satisfy the following conditions (remembering $r>0$ is fixed):

$a$ is supported in some ball $B(z, r)$,

$$
\begin{gathered}
\int_{D} a(w) d V(w)=0, \\
\|a\|_{q}=|B(z, r)|^{1 / q-1} .
\end{gathered}
$$

The space $H_{q}^{1}$ consists of all functions of the form $f(z)=\sum \lambda_{j} a_{j}(z)$ where the $a_{j}$ are $q$-atoms and $\sum\left|\lambda_{j}\right|<\infty$. The norm of $f$ in $H_{q}^{1}$ is the infimum of $\sum\left|\lambda_{j}\right|$ over all such representations of $f$. Note that all atoms satisfy $\|a\|_{1} \leq 1$.

5.8. Lemma. For $q \geq 1$, the Bergman projection $P$ is bounded from $H_{q}^{1}$ to the Bergman space $A^{1}$.

Proof. It suffices to show that $\|P a\|_{1} \leq C$ for all atoms $a$ with a constant $C$ independent of $a$. Let $a$ be an atom supported in $B(\lambda, r)$. Because of the cancellation property of an atom, we have

$$
P a(z)=\int_{D} K(z, w) a(w) d V(w)=\int_{D}[K(z, w)-K(z, \lambda)] a(w) d V(w) .
$$

Note that for $w \in B(\lambda, r)$,

$$
\begin{gathered}
|\rho(w)| \sim|\rho(z)|, \quad|w-\lambda| \leq C|\rho(\lambda)|^{1 / 2}, \\
\left|\sum \frac{\partial \rho(\lambda)}{\partial z_{j}}\left(w_{j}-\lambda_{j}\right)\right| \leq C|\rho(\lambda)| .
\end{gathered}
$$

A direct calculation yields that

$$
\begin{aligned}
|\Psi(z, w)-\Psi(z, \lambda)| & \leq C\left[|\rho(\lambda)|^{1 / 2}(|z-w|+|z-\lambda|)+|\rho(\lambda)|\right] \\
& \leq C\left[|\rho(\lambda)|^{1 / 2}\left(|\Psi(z, w)|^{1 / 2}+|\Psi(z, \lambda)|^{1 / 2}\right)+|\rho(\lambda)|\right] .
\end{aligned}
$$

By C. Fefferman's formula for the Bergman kernel [Fe], it follows that

$$
\begin{aligned}
|K(z, w)-K(z, \lambda)| \leq & C\left[\frac{|\rho(\lambda)|}{|\Psi(z, \lambda)|^{n+2}}+\frac{|\rho(\lambda)|^{1 / 2}}{|\Psi(z, \lambda)|^{n+3 / 2}}\right] \\
& +C\left[\frac{|\rho(w)|}{|\Psi(z, w)|^{n+2}}+\frac{|\rho(w)|^{1 / 2}}{|\Psi(z, w)|^{n+3 / 2}}\right] \\
\leq & C\left[\frac{|\rho(\lambda)|^{1 / 2}}{|\Psi(z, \lambda)|^{n+3 / 2}}+\frac{|\rho(w)|^{1 / 2}}{|\Psi(z, w)|^{n+3 / 2}}\right] .
\end{aligned}
$$

By Fubini's theorem and Lemma 5.6 we have

$$
\|P a\|_{1} \leq C\|a\|_{1} \int_{D} \frac{|\rho(\lambda)|^{1 / 2}}{|\Psi(z, \lambda)|^{n+3 / 2}} d V(z) \leq C^{\prime},
$$

where $C$ and $C^{\prime}$ are constants independent of $a$.

5.9. Lemma. Let $f \in A^{1}$. If the Hankel operator $H_{\bar{f}}$ is bounded from $A^{1}$ to $L^{1}$, then $f$ is in the Bloch space $\mathscr{B}$ and $|f(z)-f(w)| \leq C \beta(z, w)$.

Proof. We have already seen that the boundedness of $H_{\bar{f}}$ implies that $f \in$ $B D A_{1}$ (Theorem 5.1). That is to say, there is a constant $C$ such that for any 
ball $B=B(z, r)$ there is an analytic function $h$ such that $\int_{B}|\bar{f}-h| d V \leq C|B|$. Now the same will be true if $B$ is replaced with a polydisk with radius $c|\rho(z)|$ in the complex direction normal to the boundary and radii $c \sqrt{|\rho(z)|}$ in the complex directions parallel to the boundary. It is straightforward to extend the inequality $\int_{\mathbb{D}}|\bar{f}-\bar{f}(0)| d A \leq C \int_{\mathbb{D}}|\bar{f}-h| d A$ from the disk to a polydisk. (That is, a constant is nearly the best analytic approximant to $\bar{f}$ in $L^{1}$.) Thus $f \in B M O_{1}$. And we easily deduce from this (see [Lil] or even the remarks at the end of [Lu2]) that $f \in \mathscr{B}$ and that $|f(z)-f(w)| \leq C \beta(z, w)$. This gives our conclusion.

5.10. Theorem. Let $f \in A^{1}$. Then $H_{\bar{f}}$ is bounded from $A^{1}$ to $L^{1}$ if and only if

$$
\left(\log \frac{1}{|\rho(z)|}\right)|\partial f(z)| \cdot|\rho(z)| \in L^{\infty} .
$$

Proof. We need only prove the necessity. By Lemma 5.8, if $H_{\bar{f}}$ is bounded, then $H_{\bar{f}} P$ is bounded from $H_{2}^{1}$ to $L^{1}$. Thus $\left\|H_{\bar{f}} P a\right\|_{1} \leq C$ for all atoms $a$. Writing $B=B(\lambda, r)$ for the Bergman metric ball that supports $a$ (as in the definition of atom), it is easy to see that $H_{\bar{f}} \mathrm{~Pa}$ may be written

$$
\begin{aligned}
H_{\bar{f}} P a(z)= & \int_{D}(\bar{f}(z)-\bar{f}(w)) K(z, w) a(w) d V(w) \\
= & \int_{B}\left(\bar{f}(z)-\bar{f}_{B}\right) K(z, w) a(w) d V(w) \\
& -\int_{B}\left(\bar{f}(w)-\bar{f}_{B}\right) K(z, w) a(w) d V(w) \\
= & I_{1}(z)+I_{2}(z) .
\end{aligned}
$$

Note that we have the following for $I_{1}(z)$ :

$$
I_{1}(z)=\left(\bar{f}(z)-\bar{f}_{B}\right) \int_{B}(K(z, w)-K(z, \lambda)) a(w) d V(w) .
$$

We claim that $\left\|I_{1}\right\|_{1} \leq C$. By estimates in [Li1], there is a constant $\delta>0$ such that when $|z-w| \leq \delta$ then for any $\varepsilon$ with $0<\varepsilon<1 /(4 n+4)$ we have

$$
\beta(z, w) \leq C_{\varepsilon}\left(|\Psi(z, w)|^{2} /|\rho(z) \rho(w)|\right)^{\varepsilon} .
$$

Moreover we always have

$$
\beta(z, w) \leq C[|\log | \rho(z)||+|\log | \rho(w)||],
$$

and when $|z-w|>\delta$ we have

$$
|\Psi(z, w)| \geq 1 / C>0 .
$$

From Lemma 5.9 we have $\left|f(z)-f_{B}\right| \leq \beta+r$. From this and the proof of Lemma 5.8 we have

$$
\left\|I_{1}\right\|_{1} \leq C\|a\|_{1} \int_{D}(\beta(z, \lambda)+r)\left[\frac{|\rho(\lambda)|^{1 / 2}}{|\Psi(z, \lambda)|^{n+3 / 2}}+\frac{|\rho(w)|^{1 / 2}}{|\Psi(z, w)|^{n+3 / 2}}\right] d V(z) .
$$

Finally, (21), (22), (23), $\beta(z, \lambda) \leq \beta(z, w)+r$, and the usual estimates (Lemma 5.6) show that $\left\|I_{1}\right\|_{1} \leq C$. 
Turning to $I_{2}$, we write it as

$$
\begin{aligned}
I_{2}(z)= & \int_{B}\left(\bar{f}_{B}-\bar{f}(w)\right)(K(z, w)-K(z, \lambda)) a(w) d V(w) \\
& +K(z, \lambda) \int_{B}\left(\bar{f}_{B}-\bar{f}(w)\right) a(w) d V(w) \\
= & J_{1}(z)+J_{2}(z) .
\end{aligned}
$$

Using the estimates above, we obtain

$$
\left\|J_{1}\right\|_{1} \leq C\|a\|_{1} \int_{D}(\beta(w, \lambda)+r)\left[\frac{|\rho(\lambda)|^{1 / 2}}{|\Psi(z, \lambda)|^{n+3 / 2}}+\frac{|\rho(w)|^{1 / 2}}{|\Psi(z, w)|^{n+3 / 2}}\right] d V(z),
$$

and again obtain $\left\|J_{1}\right\|_{1} \leq C$. We are down to the following: Assuming $H_{\bar{f}}$ is bounded, we may conclude that $\left\|J_{2}\right\|_{1} \leq C$ with a constant $C$ depending only on $f$ (and of course $D$ ). Thus

$$
\left\|J_{2}\right\|_{1}=\|K(\cdot, \lambda)\|_{1}\left|\int_{B}\left(\bar{f}(w)-\bar{f}_{B}\right) a(w) d V(w)\right| \leq C
$$

holds for all atoms $a$. We may suppose $f$ is not constant. Now $f \in \mathscr{B}$ implies ([Li1]) that $0<M_{\lambda} \stackrel{\text { def }}{=}|B|^{-1} \int_{B}\left|f(w)-f_{B}\right|^{2} d V(w) \leq C$ for some constant $C$ independent of $\lambda$. Thus $a_{\lambda}(z) \stackrel{\text { def }}{=}|B|^{-1} \chi_{B}(z)\left(f(z)-f_{B}\right) / M_{\lambda}^{1 / 2}$ is a 2-atom. Since $\|K(\cdot, \lambda)\|_{1} \sim|\log | \rho(\lambda)||$, it follows from (24) that

$$
|\log | \rho(\lambda)||\left[\frac{1}{|B(\lambda, r)|} \int_{B(\lambda, r)}\left|f(w)-f_{B}\right|^{2} d V(w)\right]^{1 / 2} \leq C .
$$

It was proved in [Lil] that

$$
|\rho(\lambda) \partial f(\lambda)| \leq C\left[\frac{1}{|B(\lambda, r)|} \int_{B(\lambda, r)}\left|f(w)-f_{B}\right|^{2} d V(w)\right]^{1 / 2} .
$$

Therefore $\log |\rho(z)||\rho(z)||\partial f(z)| \in L^{\infty}$.

If the necessary conditions of Theorem 5.1 were sufficient, this would contradict Theorem 5.10. The following example will show that the conditions of Theorem 5.2 are not necessary.

Example. Let us specialize to the unit disk $\mathbb{D}$, though the example is easily generalized to any domain $D$. Let $S$ be a square centered at the origin and compactly contained in $\mathbb{D}$ and let $M \geq 1$ be an integer. Divide $S$ into $2 M$ equal vertical stripes. Let $\psi$ be a function which is constant on each stripe, takes there only the values \pm 1 , does not take the same value on two stripes with a common boundary and is 0 off $S$. It is trivial to estimate for all $f \in C^{1}$

$$
\left|\int_{\mathbb{D}} f \psi d A\right| \leq C \frac{\sup _{z \in S}|\nabla f(z)|}{M} \leq C_{S} \frac{\|f\|_{1}}{M}, \quad \text { if } f \in A^{1}
$$

with the $C$ and $C_{S}$ independent of $M$. Moreover,

$$
\int_{S}|\psi-h| d A>\delta
$$

for all $h \in \mathscr{H}$ with $\delta$ independent of $M$ and $h$. 
Let $B_{j}=B\left(w_{j}, r\right)$ be a sequence of disjoint Bergman metric disks, and let $M_{j}$ be a sequence of integers. Let $S_{j}$ be the square inside $B_{j}$ obtained as the image of $S$ under the dilation and translation taking $\mathbb{D}$ to $B_{j}$. Let $\psi_{j}$ be defined on $B_{j}$ in the same way that $\psi$ is defined on $\mathbb{D}$, using the integer $M_{j}$ for $M$. Finally, let $\varphi$ denote the function which is zero off $\bigcup_{j} B_{j}$ and equal to $\psi_{j}$ on each $B_{j}$. It is clear from (26) that $\varphi$ does not satisfy the sufficient condition (b) of Theorem 5.2. In fact,

$$
\frac{1}{\left|B_{j}\right|} \inf _{h \in \mathscr{R}} \int_{B_{j}}|\varphi-h| d A
$$

does not tend to 0 and so $\left|\log \left(1-\left|z_{j}\right|^{2}\right)\right|$ times it cannot be bounded. However, the following estimates show that $P M_{\varphi}$ is bounded from $A^{1}$ to $L^{1}$ for some choice of the sequence $M_{j}$, and therefore $H_{\varphi}=M_{\varphi}-P M_{\varphi}$ is also bounded. For $g \in A^{1}$

$$
\begin{aligned}
\left|P M_{\varphi} g(z)\right| & \leq \sum\left|\int_{B_{j}} \psi_{j}(w) g(w) K(z, w) d A(w)\right| \\
& \leq C \sum r_{j}^{3} \frac{\sup _{w \in S_{j}}\left|g^{\prime}(w) K(z, w)+g(w) \partial_{w} K(z, w)\right|}{M_{j}} \\
& \leq C \sum \frac{1}{M_{j}} \int_{B_{j}}|g| d A\left[\left|K\left(z, w_{j}\right)\right|+r_{j}\left|\nabla_{w} K\left(z, w_{j}\right)\right|\right],
\end{aligned}
$$

by $(25)$, where $r_{j} \sim\left(1-\left|w_{j}\right|\right)$ is the Euclidean radius of $B_{j}$. Since $K(\cdot, w)$ and $\nabla_{w} K(\cdot, w)$ satisfy $\left\|K(\cdot, w)+(1-|w|)\left|\nabla_{w} K(\cdot, w)\right|\right\|_{1} \leq C(1+|\log (1-|w|)|)$, it suffices to choose $M_{j} \geq\left(1+\left|\log \left(1-\left|w_{j}\right|\right)\right|\right)$ to make $\left\|P M_{\varphi} g\right\|_{1} \leq C\|g\|_{1}$.

\section{THE PROOF OF LEMMA 3.1}

Recall Lemma 5.3 from $\S 5$. We shall use it again to construct another kernel $L(z, w)$ for the solution of the $\bar{\partial}$-equation. One difference between this new one and the $L^{(N)}(z, w)$ already constructed is that the factor $|z-w|^{2(n-k)}$ in the denominator is replaced by an expression $\|z-w\|_{A}^{2(n-k)}$ where $\|\cdot\|_{A}^{2}$ is a positive definite quadratic form whose behavior is better suited to the geometry of a strongly pseudoconvex domain (and to our estimates). Following [BA], this necessitates a different choice of the form $s$ from the one defined prior to Lemma 5.4. Also, we will choose a slightly different change of variables from the one used in [Li2] and [DH]. The construction of $L$ below first appeared in [Li3].

Let us write

$$
G(z, w)=F(w, z)=\sum_{j=1}^{n} \rho_{j}(z)\left(z_{j}-w_{j}\right)-\frac{1}{2} \sum_{j=1}^{n} \sum_{k=1}^{n} \rho_{j k}(z)\left(z_{j}-w_{j}\right)\left(z_{k}-w_{k}\right)
$$

where $F(z, w)$ is the Levi polynomial for $D$. Here are a few simple observations that we will need later.

$$
\begin{aligned}
|G(z, w)+F(z, w)| & =\left|\sum_{j=1}^{n}\left(\rho_{j}(w)-\rho_{j}(z)\right)\left(w_{j}-z_{j}\right)+O\left(|z-w|^{2}\right)\right| \\
& \leq C|z-w|^{2},
\end{aligned}
$$


and if $\delta>0$ is as in Lemma 5.3 and $|z-w|<\delta$, then

$$
\begin{aligned}
|G(z, w)| & \leq C\left(|F(z, w)|+|z-w|^{2}\right) \\
& \leq C\left(|\operatorname{Im} F(z, w)|+|\operatorname{Re} F(z, w)+| \rho(w)||+|\rho(w)|+|z-w|^{2}\right) \\
& \leq C(|\operatorname{Im} F(z, w)|+|\operatorname{Re} F(z, w)+| \rho(w)||) \leq C|\Psi(z, w)| .
\end{aligned}
$$

Similarly, $|F(z, w)| \leq C|\Psi(z, w)|$ and $|\rho(z)|+|\rho(w)|+|z-w|^{2} \leq C|\Psi(z, w)|$ in $|z-w|<\delta$.

6.1. Lemma. Let $\rho$ and $G$ be as above. Then there exist constants $\delta$ and $C$ such that for any $z \in D$ with $|\rho(z)| \leq \delta$, in $|w-z|<\delta$ we can perform a smooth change of variables $t=t(w)=\left(t_{1}, t_{2}, \ldots, t_{n}\right)$ with the following properties:

(a) $t_{1}(w)=G(z, w)$.

(b) $|z-w| / C \leq|t(w)| \leq C|z-w|$ for $w$ in $|w-z|<\delta$.

(c) $1 / C \leq|\partial t / \partial w| \leq C$ for $w$ in $|w-z|<\delta$, where $\partial t / \partial w$ denotes the Jacobian of $t$.

Remark. It is standard (see [R] or [DH]) to change coordinates with a $\tau=\tau(w)$ where $\operatorname{Re} \tau_{1}(w)=\rho(w)-\rho(z)$ (or simply $\operatorname{Re} \tau_{1}(w)=\rho(w)$ ) and $\operatorname{Im} \tau_{1}(w)=$ $\operatorname{Im} G(z, w)$. These have the advantage that level sets of $\rho$ are mapped to hyperplanes, but the special nature of the integrals we will need to estimate seems to require us to use the slightly different coordinates of the lemma. (The real parts of $t_{1}$ and $\tau_{1}$ differ only by $O\left(|z-w|^{2}\right)$ near $z$.)

Now choose a smooth function $\chi$ on $\mathbb{C}^{n} \times \mathbb{C}^{n}$ such that $0 \leq \chi \leq 1$ and

$$
\chi(z, w)= \begin{cases}1 & \text { if }|z-w| \leq \delta / 2 \\ 0 & \text { if }|z-w| \geq \delta\end{cases}
$$

and write

$$
\begin{aligned}
G_{1}(z, w) & =\chi G(z, w)+(1-\chi)|z-w|^{2}, \\
\|z-w\|_{A}^{2} & =\left|G_{1}(z, w)\right|^{2}+|\rho(z)||z-w|^{2},
\end{aligned}
$$

and

$$
\begin{aligned}
s_{j}(z, w)= & \overline{G_{1}(z, w)}\left[\chi(z, w)\left(\rho_{j}(z)-\frac{1}{2} \sum_{k=1}^{n} \rho_{j k}\left(z_{k}-w_{k}\right)\right)\right. \\
& \left.+(1-\chi(z, w))\left(\overline{z_{j}-w_{j}}\right)\right] \\
& +|\rho(z)|\left(\overline{z_{j}-w_{j}}\right) .
\end{aligned}
$$

It is easy to calculate that $\|z-w\|_{A}^{2}=\sum s_{j}(z, w)\left(z_{j}-w_{j}\right)$, that $\left|s_{j}\right| \leq C|z-w|$ uniformly for $z, w \in \bar{D}$, and that for $z$ in any compact subset $\Omega$ of $D$, $\|z-w\|_{A}^{2} \geq C^{\prime}|z-w|^{2}$ uniformly for $w \in \bar{D}$. (The constant $C^{\prime}$ is allowed to depend on the compact set $\Omega$.) Taken together, these statements say that the $s_{j}$ satisfy condition (1) in [BA].

6.2. Lemma [Li3]. The quantities $G$ and $\|\cdot\|_{A}$ satisfy the following inequalities.

(a) If $|z-w|<\delta / 2$ then $\|z-w\|_{A}^{2} \geq(1 / C)\left(|G(z, w)|^{2}+|\rho(w)||z-w|^{2}\right)$. 
(b) If $|z-w| \geq \delta / 2$ then $\|z-w\|_{A}^{2} \geq|\rho(w)| / C$.

Following B. Berndtsson and M. Andersson [BA] we define

$$
\begin{aligned}
& s(z, w)=\sum_{j=1}^{n} s_{j}(z, w) d w_{j}, \quad h(z, w)=\sum_{j=1}^{n} h_{j}(z, w) d w_{j}, \\
& q(z, w)=\bar{\partial}_{w} j(z, w) / \rho(w)-\bar{\partial} \rho(w) \wedge h(z, w) / \rho(w)^{2},
\end{aligned}
$$

and

$$
L(z, w)=C_{n} \sum_{0}^{n-1} \gamma_{k}\left[\frac{|\rho(w)|}{\Psi(z, w)}\right]^{k+n+1} \frac{s(z, w) \wedge q(z, w)^{k} \wedge\left(\bar{\partial}_{w} s\right)^{n-k-1}}{\|z-w\|_{A}^{2(n-k)}},
$$

where $\gamma_{k}$ are constants defined in [BA, equation (20), p. 102] by letting $N=$ $n+1$ and $C_{n}=(-1)^{n(n+1) / 2} /(n-1)$ !. Also, $h$ is the same $h$ from Lemma 5.3 and $q$ is the same as in $\S 5$.

6.3. Lemma [BA, pp. 103-104]. If $\varphi$ is a $\bar{\partial}$-closed $(0,1)$ form with coefficients in $C^{1}(\bar{D})$, then

$$
u(z)=\int_{D} \varphi \wedge L(z, w)
$$

is a solution to the equation $\bar{\partial} u=\varphi$.

Remark. In [BA], this result was proved for strictly convex domains by letting

$$
s_{j}(z, w)=\left[\sum_{k=1}^{n} \rho_{k}(z)\left(\overline{z_{k}-w_{k}}\right)\right] \rho_{j}(z)+|\rho(z)|\left(\overline{z_{j}-w_{j}}\right)
$$

and

$$
h_{j}(z, w)=\rho_{j}(w),
$$

for $1 \leq j \leq n$. As indicated in [BA, p. 104], an application of the same arguments yields the result stated here.

Remark. By a standard argument (e.g., see [DH, p. 453]), Lemma 6.3 may be extended to $\bar{\partial}$-closed $(0,1)$ forms with coefficients in $C^{1}(D) \cap L^{1}(D)$.

It is easy to check the following for $|z-w|<\delta / 2$.

$$
\begin{aligned}
s(z, w)= & \overline{G(z, w)}\left[\sum_{1}^{n} \rho_{j}(z) d w_{j}+\beta_{1}(z, w)\right]+|\rho(z)| \sum_{1}^{n}\left(\overline{z_{j}-w_{j}}\right) d w_{j}, \\
\bar{\partial}_{w} s(z, w)= & \bar{\partial}_{w} \overline{G(z, w)} \wedge\left[\sum_{1}^{n} \rho_{j}(z) d w_{j}+\beta_{1}(z, w)\right]+\rho(z) \sum_{1}^{n} d \bar{w}_{j} \wedge d w_{j} \\
= & {\left[-\sum_{1}^{n} \bar{\rho}_{j} d \bar{w}_{j}+\overline{\beta_{2}(z, w)}\right] \wedge\left[\sum_{1}^{n} \rho_{j}(z) d w_{j}+\beta_{1}(z, w)\right] } \\
& +\rho(z) \sum_{1}^{n} d \bar{w}_{j} \wedge d w_{j},
\end{aligned}
$$

where $\beta_{m}, m=1,2$, are $(1,0)$ forms with $\left|\beta_{m}(z, w)\right| \leq C|z-w|$ and $\bar{\partial}_{w} \beta_{1}=0$. 
Recall from $\S 5$ that

$$
q(z, w)^{k}=\frac{\rho(w)\left(\bar{\partial}_{w} h(z, w)\right)^{k}-k \bar{\partial} \rho(w) \wedge h(z, w) \wedge\left(\bar{\partial}_{w} h(z, w)\right)^{k-1}}{\rho(w)^{k+1}} .
$$

Also, for $|z-w|<\delta / 2$ and $k<n-1$,

$$
\left(\bar{\partial}_{w} s\right)^{n-k-1}=\left[\rho a+(n-k-1) \bar{\partial}_{w} \bar{G} \wedge \bar{s}\right] \wedge(\rho a)^{n-k-2}
$$

where

$$
a(z, w)=\sum_{1}^{n} d \bar{w}_{j} \wedge d w_{j}, \quad \bar{s}(z, w)=\sum_{1}^{n} \rho_{j}(z) d w_{j}+\beta_{1}(z, w) .
$$

It follows that we may write the combination $s(z, w) \wedge\left(\bar{\partial}_{w} s(z, w)\right)^{n-k-1}$ that appears in the formula for $L(z, w)$ as $s \wedge\left(\bar{\partial}_{w} s\right)^{n-k-1}=\rho(z)^{n-k-1}\left\{\bar{G} \tilde{s} \wedge a+g_{1} \wedge[\rho(z) a+(n-k-1) \overline{\partial G} \wedge \bar{s}]\right\} \wedge a^{n-k-2}$, where $g_{1}(z, w)=\sum_{1}^{n}\left(\overline{z_{j}-w_{j}}\right) d w_{j}$.

This gives us the following formula for $L(z, w)$ :

$$
L(z, w)=C_{n} \sum_{k=0}^{n-1} \gamma_{k} L_{k}(z, w)
$$

with

$$
\begin{aligned}
& L_{k}(z, w) \\
& =\frac{\rho(z)^{n-k-1} \rho(w)^{n}}{\Psi(z, w)^{n+k+1}\|z-w\|_{A}^{2(n-k)}}\left[\rho(w)\left(\bar{\partial}_{w} h\right)^{k}-k \bar{\partial} \rho(w) \wedge h \wedge\left(\bar{\partial}_{w} h\right)^{k-1}\right] \\
& \quad \wedge\left\{\bar{G} \tilde{s} \wedge a+g_{1} \wedge[\rho(z) a+(n-k-1) \bar{\partial} \bar{G} \wedge \tilde{s}]\right\} \wedge a^{n-k-2},
\end{aligned}
$$

except that when $k=0$ the first bracket becomes simply $\rho(w)$, and when $k=n-1$ the entire expression on the last line becomes $\bar{G} \tilde{s}+\rho g_{1}$ which equals $s(z, w)$.

For convenience we will use $\mathscr{E}_{m}(z, w)$ to denote smooth forms on $\bar{D} \times \bar{D}$ satisfying $\left|\mathscr{E}_{m}(z, w)\right| \leq C|z-w|^{m}$. The actual form represented by $\mathscr{E}_{m}$ may change from one instance to another, even on the same line.

Note the following estimates:

$$
\begin{aligned}
\partial_{w} G_{1}(z, w) & =\partial_{w} G(z, w)=-\partial \rho(w)+\mathscr{E}_{1}, \\
\tilde{s}(z, w) & =\sum \rho_{j}() d w_{j}+\beta_{1}(z, w)=\partial \rho(w)+\mathscr{E}_{1}, \\
h(z, w) & =\partial \rho(w)+\mathscr{E}_{1}+\rho(w) \mathscr{E}_{0} .
\end{aligned}
$$

It follows that

$$
\begin{gathered}
\bar{\partial}_{w} \overline{G_{1}(z, w)}=\bar{\partial} \rho(w)+\mathscr{E}_{1}(z, w), \\
\tilde{s}(z, w) \wedge h(z, w)=\mathscr{E}_{1}(z, w)+\mathscr{E}_{0}(z, w) \rho(w), \\
\bar{\partial}_{w} \bar{G}_{1} \wedge \tilde{s}(z, w) \wedge \bar{\partial} \rho(w) \wedge h(z, w) \\
=\bar{\partial} \rho(w) \wedge \mathscr{E}_{1}(z, w)\left[\mathscr{E}_{1}(z, w)+\rho(w) \mathscr{E}_{0}(z, w)\right] .
\end{gathered}
$$


Thus we have

(27)

$$
\begin{aligned}
& L_{k}(z, w) \\
& =\frac{\rho(z)^{n-k-1} \rho(w)^{n}}{\Psi(z, w)^{n+k+1}\|z-w\|_{A}^{2(n-k)}}\left\{\left[(n-k-1) \mathscr{E}_{2}+\rho(z) \mathscr{E}_{1}+\bar{G} \mathscr{E}_{0}\right] \wedge \rho(w)\right. \\
& \left.\quad+\left[k\left(\rho(z) \mathscr{E}_{1}+\bar{G} \mathscr{E}_{1}\right)+(n-k-1)\left(\rho(w) \mathscr{E}_{1}+k \mathscr{E}_{3}\right)\right] \wedge \bar{\partial} \rho(w)\right\} .
\end{aligned}
$$

(We keep the $k$ and the $n-k-1$ before certain terms rather than absorb them in the $\mathscr{E}_{m}$, because these terms vanish in the extreme cases $k=0$ or $k=n-1$.)

In the next lemma we give estimates for the solution of the $\bar{\partial}$-equation. It seems to us that the standard integral representations and estimates [R, DH] do not work in our case. The following estimates may therefore be of some independent interest.

6.4. Lemma. If $\varphi$ is a $\bar{\partial}$-closed $(0,1)$ form with coefficients in $C^{1}(D) \cap L^{1}(D)$ and the expression $|\varphi \wedge \bar{\partial} \rho||\rho|^{1 / 2}+|\rho \varphi|$ belongs to $L^{\infty}(D)$, then the solution

$$
u(z)=\int_{D} \varphi(w) \wedge L(z, w)
$$

of the $\bar{\partial}$-equation $\bar{\partial} u=\varphi$ has the form $u(z)=u_{1}(z)+u_{2}(z)$, where

$$
\left\|u_{1}\right\|_{\infty}+\left\|\rho \nabla u_{2}\right\|_{\infty} \leq C\left\||\rho \varphi|+|\rho|^{1 / 2}|\varphi \wedge \bar{\partial} \rho|\right\|_{\infty}
$$

and

$$
\left|\partial u_{2}(z) \wedge \partial \rho(z)\right|+\left|\bar{\partial} u_{2}(z) \wedge \bar{\partial} \rho(z)\right| \leq C|\rho(z)|^{-1 / 2}\left\||\rho \varphi|+|\rho|^{1 / 2}|\varphi \wedge \bar{\partial} \rho|\right\|_{\infty} .
$$

Proof. We may assume without loss of generality that the $L^{\infty}$ norm of $|\varphi \wedge \bar{\partial} \rho||\rho|^{1 / 2}+|\rho \varphi|$ is 1 . By Lemmas 5.3 and (6.2), if $|z-w| \geq \delta / 2$ then $\|\varphi \wedge L\|_{\infty} \leq C$. If $|\rho(z)| \geq \delta$ and $|z-w|<\delta$ then $|\varphi(w) \wedge L(z, w)| \leq$ $C /|z-w|^{2 n-1}$ and a similar estimate holds for $|\rho(w)|>\delta$. Thus the portion of the integral in (28) over $|w-z|>\delta / 2$ is bounded independently of $z$ and the portion of the integral over $|w-z|<\delta$ is bounded for $|\rho(z)|>\delta$. Thus, it suffices to obtain estimates when $z$ is close to the boundary of $D$ and we need only estimate the integral over $|w-z|<\delta / 2$.

Now write

$$
v_{k}(z)=\int_{D} \varphi(w) \wedge L_{k}(z, w) .
$$

We begin by showing that for $0 \leq k \leq n-2, v_{k}$ is actually bounded. It is easy to check from (27) that for such $k,\left|\varphi(w) \wedge L_{k}(z, w)\right|$ is dominated by the sum of the two terms

$$
\frac{|\rho(z)|^{n-k-1}|\rho(w)|^{n-1 / 2}}{\|z-w\|_{A}^{2(n-k)}|\Psi(z, w)|^{n+k+1}}\left(|G(z, w)|+|\rho(w)|+|\rho(z)|+|z-w|^{2}\right)|z-w|
$$

and

$$
\frac{|\rho(z)|^{n-k-1}|\rho(w)|^{n}}{\|z-w\|_{A}^{2(n-k)}|\Psi(z, w)|^{n+k+1}}\left(|G(z, w)|+|\rho(z)||z-w|+|z-w|^{2}\right) .
$$

Note that, from Lemma 5.3 when $|z-w| \leq \delta$ and $|\rho(z)|<\delta$,

$$
|\Psi(z, w)| \geq(\operatorname{Re} F(z, w)+|\rho(w)|) / C \geq\left(|\rho(z)|+|\rho(w)|+|z-w|^{2}\right) / C .
$$


Recall also that $|\Psi(z, w)| \geq|G(z, w)| / C$. Thus the terms above are dominated by

$$
\begin{aligned}
& \frac{|\rho(z)|^{n-k-1}|G(z, w)|}{\|z-w\|_{A}^{2(n-k)}|\Psi(z, w)|^{k+1}}+\frac{|\rho(z)|^{n-k-1}|\rho(w)|^{n-1 / 2}}{\|z-w\|_{A}^{2(n-k)}|\Psi(z, w)|^{n+k}}|z-w| \\
& \quad=I_{1}(z, w)+I_{2}(z, w) .
\end{aligned}
$$

Using the coordinate system of Lemma 6.1 with $t=\left(t_{1}, t_{2}, \ldots, t_{n}\right)$, it follows that

$$
\int_{|z-w|<\delta} I_{1}(z, w) d V(w) \leq C \int_{|t|<1} \frac{|\rho(z)|^{n-k-1}\left|t_{1}\right| d t}{\left(\left|t_{1}\right|^{2}+|\rho(z)||t|^{2}\right)^{n-k}\left(|\rho(z)|+|t|^{2}\right)^{k+1}} .
$$

Changing to polar coordinates with $s=\left|t_{1}\right|$ and $r=\left|t^{\prime}\right|$, where $t^{\prime}=\left(t_{2}, \ldots, t_{n}\right)$, we have

(29)

$$
\begin{aligned}
\int_{|z-w|<\delta} I_{1}(z, w) d V(w) & \leq C \int_{0}^{1} \int_{0}^{1} \frac{|\rho(z)|^{n-k-1} s^{2} d s r^{2 n-3} d r}{\left(s^{2}+|\rho(z)| r^{2}\right)^{n-k}\left(|\rho(z)|+r^{2}\right)^{k+1}} \\
& \leq C \int_{0}^{1} \int_{0}^{1} \frac{|\rho(z)|^{-1 / 2} s d s r^{2 n-3} d r}{\left(s^{2} /|\rho(z)|+r^{2}\right)^{n-k-1 / 2}\left(|\rho(z)|+r^{2}\right)^{k+1}} \\
& \leq C \int_{0}^{1} \frac{|\rho(z)|^{1 / 2} r^{2 k} d r}{\left(|\rho(z)|+r^{2}\right)^{k+1}} \leq C \int_{0}^{1} \frac{|\rho(z)|^{1 / 2} d r}{|\rho(z)|+r^{2}} \leq C .
\end{aligned}
$$

The passage from the next to last line to the last line requires $k \leq n-2$. To estimate the integral of $I_{2}$ we use Lemma 6.2(a) and obtain

$$
\begin{aligned}
\|z-w\|_{A}^{2(n-k)} & \geq c\|z-w\|_{A}^{2(n-k)-1}\left(|G(z, w)|^{2}+|\rho(w)||z-w|^{2}\right)^{1 / 2} \\
& \geq c\|z-w\|_{A}^{2(n-k)-1}|\rho(w)|^{1 / 2}|z-w| .
\end{aligned}
$$

Thus

$$
I_{2}(z, w) \leq \frac{C|\rho(z)|^{n-k-1}}{\|z-w\|_{A}^{2(n-k)-1}|\Psi(z, w)|^{k+1}} .
$$

Using the same coordinate system as before, we obtain

$$
\begin{aligned}
\int_{|z-w|<\delta} I_{2}(z, w) d V(w) & \leq C \int_{|t|<1} \frac{|\rho(z)|^{n-k-1} d t}{\left(\left|t_{1}\right|^{2}+|\rho(z)||t|^{2}\right)^{n-k-1 / 2}\left(|\rho(z)|+|t|^{2}\right)^{k+1}} \\
& \leq C \int_{|t|<1} \frac{|\rho(z)|^{-1 / 2} d t}{\left(\left|t_{1}\right|^{2} /|\rho(z)|+|t|^{2}\right)^{n-k-1 / 2}\left(|\rho(z)|+|t|^{2}\right)^{k+1}} .
\end{aligned}
$$

After a change to polar coordinates, the last integral is dominated by the second line of (29). Thus $\int_{|z-w|<\delta} I_{2}(z, w) d V(w) \leq C$. Therefore $v_{k}(z) \leq C$ for all $z \in D$ and $0 \leq k \leq n-2$. Thus we can write $u_{1}=v_{1}+\cdots+v_{n-2}$, and $u_{1}$ is bounded as required.

We set $u_{2}=v_{n-1}$ and estimate the gradient of $u_{2}$. Note that $\partial_{z} G=\partial \rho(z)+$ $\mathscr{E}_{1}$ and $\bar{\partial}_{z} G=\mathscr{E}_{1}$. Therefore

$$
\left|\partial_{z}\left(\|z-w\|_{A}^{2}\right) \wedge \partial \rho(z)\right|+\left|\bar{\partial}_{z}\left(\|z-w\|_{A}^{2}\right) \wedge \bar{\partial} \rho(z)\right| \leq C(|\rho(z)|+|G|)|z-w|,
$$

and

$$
\left|\partial_{z} \Psi(z, w) \wedge \partial \rho(z)\right| \leq C(|z-w|+|\Psi(z, w)|) .
$$


From the expression for $L_{n-1}$ in (27) and the definition of $\|z-w\|_{A}^{2}$ it follows that

$$
\begin{aligned}
& \left|\partial u_{2}(z) \wedge \partial \rho(z)\right|+\left|\bar{\partial} u_{2}(z) \wedge \bar{\partial} \rho(z)\right| \\
& \leq C \int_{D}\left[\frac{|\rho(z)|+|G|+|z-w|^{2}}{\|z-w\|_{A}^{2}\left|\Psi(z, w)^{2 n}\right|}+\frac{(|\rho(z)|+|G|)|z-w|^{2}}{\|z-w\|_{A}^{2}\left|\Psi(z, w)^{2 n+1}\right|}\right]|\rho(w)|^{n-1 / 2} d V(w) \\
& \quad+C \int_{D}\left[\frac{|\rho(z)|^{1 / 2}+|z-w|}{\|z-w\|_{A}^{2}\left|\Psi(z, w)^{2 n}\right|}+\frac{|G||z-w|}{\|z-w\|_{A}^{2}\left|\Psi(z, w)^{2 n+1}\right|}\right]|\rho(w)|^{n} d V(w) \\
& =J_{1}+J_{2}
\end{aligned}
$$

where $J_{1}$ contains the terms which have neither the factor $|z-w|$ nor $|G|$ in their numerators and $J_{2}$ contains the remaining terms.

From previous estimates

$$
|G(z, w)|+|\rho(z)|+|\rho(w)|+|z-w|^{2} \leq C|\Psi(z, w)| .
$$

Using again the coordinate system of Lemma 6.1 we have

$$
\begin{aligned}
J_{1}(z) & \leq C \int_{D}\left[\frac{|\rho(z)||\rho(w)|^{n-1 / 2}}{\|z-w\|_{A}^{2}|\Psi(z, w)|^{2 n}}+\frac{|\rho(z)|^{1 / 2}|\rho(w)|^{n}}{\|z-w\|_{A}^{2}|\Psi(z, w)|^{2 n}}\right] d V(w) \\
& \leq C|\rho(z)|^{1 / 2} \int_{D} \frac{1}{\|z-w\|_{A}^{2}|\Psi(z, w)|^{n}} d V(w) \\
& \leq C|\rho(z)|^{1 / 2} \int_{|t|<1} \frac{d t}{\left(\left|t_{1}\right|^{2}+|\rho(z)||t|^{2}\right)\left(|\rho(z)|+\left|t_{1}\right|+|t|^{2}\right)^{n}}
\end{aligned}
$$

(changing to polar coordinates)

$$
\leq C|\rho(z)|^{1 / 2} \int_{0}^{1} \int_{0}^{1} \frac{r^{2 n-3} d r s d s}{\left(s^{2}+|\rho(z)| r^{2}\right)\left(|\rho(z)|+s+r^{2}\right)^{n}}
$$

(letting $\left.s=|\rho(z)| s^{\prime}, r=|\rho(z)|^{1 / 2} r^{\prime}\right)$

$$
\begin{aligned}
& \leq C \int_{0}^{\infty} \int_{0}^{\infty} \frac{|\rho(z)|^{-1 / 2} r^{2 n-3} d r^{\prime} s^{\prime} d s^{\prime}}{\left(s^{\prime 2}+r^{\prime 2}\right)\left(1+s^{\prime}+r^{\prime 2}\right)^{n}} \\
& \leq C|\rho(z)|^{-1 / 2} \int_{0}^{\infty} \int_{0}^{\infty} \frac{r^{\prime 2 n-4} d r^{\prime} d s^{\prime}}{\left(1+s^{\prime}+r^{\prime 2}\right)^{n}} \\
& \leq C|\rho(z)|^{-1 / 2} \int_{0}^{\infty} \frac{r^{\prime 2 n-4} d r^{\prime}}{\left(1+r^{\prime 2}\right)^{n-1}} \\
& \leq C|\rho(z)|^{-1 / 2} \int_{0}^{\infty} \frac{d r^{\prime}}{1+r^{\prime 2}} \leq C|\rho(z)|^{-1 / 2} .
\end{aligned}
$$

In order to estimate $J_{2}$ we use Lemma 6.2, the definition of $\|z-w\|_{A}^{2}$ and the inequality $|\rho(z)|+|\rho(w)|+|z-w|^{2} \leq C|\Psi(z, w)|$ to get

$$
J_{2} \leq C \int_{D}\left(\frac{1}{|z-w||\Psi(z, w)|^{n+1}}+\frac{1}{|\Psi(z, w)|^{n+3 / 2}}\right) d V(w) .
$$

The standard estimate given in [DH, p. 454] now yields $J_{2} \leq C|\rho(z)|^{-1 / 2}$, and we have thus proved that the gradient of $u_{2}$ in the complex tangential directions (that is, orthogonal to the gradient of $\rho$ at $z$ ) are bounded by $C|\rho(z)|^{-1 / 2}$. 
An application of similar arguments yields that

$$
\left|\partial u_{2}(z)\right|+\left|\bar{\partial} u_{2}(z)\right| \leq C /|\rho(z)|,
$$

so that the gradient of $u_{2}$ in the complex direction parallel to the gradient of $\rho$ behaves like $|\rho(z)|^{-1}$. The lemma is proved.

\section{REFERENCES}

[Ax1] S. Axler, Bergman spaces and their operators, Surveys of Some Recent Results in Operator Theory. Vol. 1 (J. B. Conway and B. B. Morell, eds.), Pitman Research Notes in Math., vol. 171, Longman Sci. Tech., Harlow, 1988, pp. 1-50.

[Ax2] - The Bergman space, the Bloch space, and commutators of multiplication operators, Duke Math. J. 53 (1986), 315-322.

[BL] F. Beatrous and S.-Y. Li, On the boundedness and compactness of operators of Hankel type, J. Funct. Anal. 111 (1993), 350-379.

[BBCZ] D. Békollé, C. A. Berger, L. A. Coburn, and Kehe Zhu, BMO in the Bergman metric on bounded symmetric domains, J. Funct. Anal. 93 (1990), 310-350.

[BCZ] C. A. Berger, L. A. Coburn, and Kehe Zhu, BMO in the Bergman spaces of the classical domains, Bull. Amer. Math. Soc. 17 (1987), 133-136.

[BA] B. Berndtsson and M. Andersson, Henkin-Ramirez formulas and weight factors, Ann. Inst. Fourier (Grenoble) 12 (1982), fasc. 3, 91-110.

[Bo] F. Bonsall, Decompositions of functions as sums of elementary functions, Quart. J. Math. Oxford Ser. (2) 37 (1986), 129-136.

[C] B. Coupet, Décomposition atomique des espaces de Bergman, Indiana Univ. Math. J. 38 (1989), 917-941.

[DH] Sh. A. Dautov and G. M. Henkin, Zeros of holomorphic functions of finite order and weighted estimates for solutions of the $\bar{\partial}$-equation, Mat. Sbornik 107 (1978), 163-174; English transl. in Math. USSR-Sb. 35 (1979), 449-459.

[Fe] C. Fefferman, The Bergman kernel and biholomorphic mappings of pseudoconvex domains, Invent. Math. 26 (1974), 1-65.

[F] J. E. Fornaess, Embedding strictly pseudoconvex domains in convex domains, Amer. J. Math. 98 (1976), 529-569.

[Go] D. Goldberg, A local version of real Hardy spaces, Duke Math. J. 46 (1979), 27-42.

[G] I. Graham, Boundary behavior of the Carathéodory and Kobayashi metrics on strongly pseudoconvex domains in $\mathbb{C}^{n}$, Trans. Amer. Math. Soc. 207 (1975), 219-240.

[KM] S. G. Krantz and Daowei Ma, Bloch functions on strongly pseudoconvex domains, Indiana Univ. Math. J. 37 (1988), 145-165.

[Li] Huiping Li, BMO, VMO and Hankel operators on the Bergman space of strongly pseudoconvex domains, J. Funct. Anal. 106 (1992), 375-408.

[Li2] _ Hankel operators on the Bergman space of strongly pseudoconvex domains, Integral Equations Operator Theory (to appear).

[Li3] _ Schatten class Hankel operators on strongly pseudoconvex domains, Proc. Amer. Math. Soc. 119 (1993), 1211-1221.

[Lu1] D. H. Luecking, A technique for characterizing Carleson measures on Bergman spaces, Proc. Amer. Math. Soc. 87 (1983), 656-660.

[Lu2] - Forward and reverse Carleson inequalities for functions in Bergman spaces and their derivatives, Amer. J. Math. 107 (1985), 85-111.

[Lu3] Characterizations of certain classes of Hankel operators on the Bergman spaces of the unit disk, J. Funct. Anal. 110 (1992), 247-271.

[R] R. M. Range, Holomorphic functions and integral representations in several complex variables, Springer-Verlag, New York, 1986. 
[V] N. Th. Varopoulos, BMO functions and the $\bar{\partial}$-equation, Pacific J. Math. 71 (1977), 221-273.

[Z] Kehe Zhu, Multipliers of BMO in the Bergman metric with applications to Toeplitz operators, J. Funct. Anal. 87 (1989), 31-50.

Department of Mathematics, SUNY at Buffalo, Buffalo, New York 14214

E-mail address: hlioriemann.math buffalo.edu

Department of Mathematics, University of Arkansas, Fayetteville, Arkansas 72701

E-mail address: luecking@comp.uark. edu 